\title{
Characteristics of Langmuir Turbulence in the Ocean Mixed Layer
}

Article

Published Version

Grant, A. L. M. and Belcher, S. E. (2009) Characteristics of Langmuir Turbulence in the Ocean Mixed Layer. Journal of Physical Oceanography, 39 (8). pp. 1871-1887. ISSN 00223670 doi: https://doi.org/10.1175/2009JPO4119.1 Available at https://centaur.reading.ac.uk/1750/

It is advisable to refer to the publisher's version if you intend to cite from the work. See Guidance on citing.

Published version at: http://ams. allenpress.com/perlserv/?request=get-abstract\&doi=10.1175\%2F2009JPO4119.1 To link to this article DOI: http://dx.doi.org/10.1175/2009JPO4119.1

Publisher: American Meteorological Society

Publisher statement: (c) Copyright [2009-08] American Meteorological Society (AMS). Permission to use figures, tables, and brief excerpts from this work in scientific and educational works is hereby granted provided that the source is acknowledged. Any use of material in this work that is determined to be "fair use" under Section 107 of the U.S. Copyright Act or that satisfies the conditions specified in Section 108 of the U.S. Copyright Act (17USC $\$ 108$, as revised by P.L. 94-553) does not require the AMS's permission. Republication, systematic reproduction, posting in electronic form, such as on a web site or in a searchable database, or other uses of this material, except as exempted by the above statement, requires written permission or a license from the AMS. Additional details are provided in the AMS Copyright Policy, available on the AMS Web site located at (http://www.ametsoc.org/) or from the AMS at 617-227-2425 or copyright@ametsoc.org.

All outputs in CentAUR are protected by Intellectual Property Rights law, 
including copyright law. Copyright and IPR is retained by the creators or other copyright holders. Terms and conditions for use of this material are defined in the End User Agreement.

\section{www.reading.ac.uk/centaur}

\section{CentAUR}

Central Archive at the University of Reading

Reading's research outputs online 


\title{
Characteristics of Langmuir Turbulence in the Ocean Mixed Layer
}

\author{
Alan L. M. Grant And Stephen E. Belcher \\ University of Reading, Reading, United Kingdom
}

(Manuscript received 12 August 2008, in final form 21 January 2009)

\begin{abstract}
This study uses large-eddy simulation (LES) to investigate the characteristics of Langmuir turbulence through the turbulent kinetic energy (TKE) budget. Based on an analysis of the TKE budget a velocity scale for Langmuir turbulence is proposed. The velocity scale depends on both the friction velocity and the surface Stokes drift associated with the wave field. The scaling leads to unique profiles of nondimensional dissipation rate and velocity component variances when the Stokes drift of the wave field is sufficiently large compared to the surface friction velocity. The existence of such a scaling shows that Langmuir turbulence can be considered as a turbulence regime in its own right, rather than a modification of shear-driven turbulence.

Comparisons are made between the LES results and observations, but the lack of information concerning the wave field means these are mainly restricted to comparing profile shapes. The shapes of the LES profiles are consistent with observed profiles. The dissipation length scale for Langmuir turbulence is found to be similar to the dissipation length scale in the shear-driven boundary layer. Beyond this it is not possible to test the proposed scaling directly using available data. Entrainment at the base of the mixed layer is shown to be significantly enhanced over that due to normal shear turbulence.
\end{abstract}

\section{Introduction}

Foam and other buoyant materials on the surface of the ocean often form lines that are almost parallel to the direction of the wind. Langmuir (1938) showed that these lines form along the convergence zones between counter-rotating vortices in the underlying water. The circulations are now generally believed to form through an instability arising from the interaction of the Stokes drift, induced by the surface waves, and the shear in the current (Craik and Leibovich 1976) through the second Craik-Leibovich (CL2) mechanism.

Skyllingstad and Denbo (1995) used the CraikLeibovich vortex force to parameterize wave-current interactions in a large-eddy simulation (LES) in a study of the effects of Langmuir circulations on mixing in the oceanic mixed layer. In these simulations the Langmuir circulations were found to dominate the vertical mixing, leading to enhanced dissipation rates that were consistent with observations (Lombardo and Gregg 1989; Anis and Moum 1992). McWilliams et al. (1997) intro-

Corresponding author address: Alan L. M. Grant, University of Reading, P.O. Box 243, Earley Gate, Reading, Berkshire RG6 6BB, United Kingdom.

E-mail: a.l.m.grant@reading.ac.uk duced the turbulent Langmuir number, $\mathrm{La}_{t}=\left(u * / u_{\mathrm{s} 0}\right)^{1 / 2}$, where $u *$ is the surface friction velocity in the water and $u_{\mathrm{s} 0}$ is the surface Stokes drift, as an important parameter describing Langmuir turbulence. Li et al. (2005) investigated the variation in turbulent statistics, particularly the nondimensional vertical velocity variance $\sigma_{w}^{2} / u_{*}^{2}$ with $\mathrm{La}_{t}$. They concluded, that for values of $\mathrm{La}_{t}$ appropriate to fully developed seas in the open ocean, Langmuir turbulence should be important. Polton and Belcher (2007) considered the transition between sheardominated turbulence and Langmuir turbulence as a function of $\mathrm{La}_{t}$ and the depth scale of the Stokes shear. In all of these studies the vertical velocity variance, normalized by $u_{*}^{2}$, was found to be much larger for Langmuir turbulence than for normal shear-driven turbulence, which is qualitatively consistent with the observations of D'Asaro (2001) and Tseng and D'Asaro (2004).

The LES studies have provided a simple conceptual picture of Langmuir turbulence. Elongated vortex structures, with alternating sign of the longitudinal vorticity are confined to the layer of Stokes shear. In the longitudinal direction the vortices have a length that is about five times that of the transverse dimension (McWilliams et al. 1997). The horizontal scale increases with depth. Polton and Belcher (2007) show that downwelling jets 
originating within the vortex layer penetrate deeply into the underlying mixed layer. Because of these jets the influence of the surface waves on the mixed layer extends below the layer of Stokes drift and Langmuir turbulence affects mixing throughout the depth of the mixed layer.

Similarity theories provide a compact way of organizing and understanding turbulence data. Holtslag and Nieuwstadt (1986) present a diagram showing the scaling regimes for the atmospheric boundary layer (ABL). Their diagram was constructed in terms of nondimensional height, $z / h$, within the boundary layer, where $h$ is the mixed layer depth, and a stability parameter, $-h / L$, where $L$ is the Obukhov length. Different scales are relevant in different regions of the diagram, so for the convective $\mathrm{ABL}$ the relevant scales are the surface buoyancy flux, $\overline{w^{\prime} b_{0}^{\prime}}$, and mixed layer depth, with the velocity scale $w_{*}=\left(\overline{w^{\prime} b_{0}^{\prime}} h\right)^{1 / 3}$, while if $-h / L$ is small, $u *$ is the relevant velocity scale.

Li et al. (2005) have constructed a regime diagram for the ocean mixed layer based on the behavior of $\sigma_{w}^{2} / u_{*}^{2}$ as a function of $\mathrm{La}_{t}$, using the Hoenikker number to characterize stability ( $\mathrm{Li}$ and Garret 1995). This diagram shows a region dominated by shear, with velocity scale $u *$, one in which convection dominates, with velocity scale $w_{*}$, and a region dominated by Langmuir turbulence. For this latter region of the diagram, Li et al. (2005) do not suggest a velocity scale.

The scaling regimes in the atmospheric boundary layer described by Holtslag and Nieuwstadt (1986) reflect the different mechanisms for generating turbulence kinetic energy (TKE), through shear or buoyancy (additionally the diagram contains information on length scales). In the Li et al. (2005) regime diagram it might be expected that the scales for Langmuir turbulence should reflect the production of turbulence through the action of the Craik-Leibovich vortex force. There have been a number of suggestions for the velocity scale of Langmuir turbulence (Plueddemann et al. 1996; Smith 1996, 1998), but at present there is no consensus as to what the appropriate velocity scale is (Thorpe et al. 2003).

The determination of the correct scaling for a turbulent flow is important in developing parameterizations to represent the effects of the turbulence in large-scale models. In first-order closure schemes of turbulent mixing, such as the $K$ profile parameterization (KPP) scheme described by Large et al. (1994), the eddy diffusivity, $K$, is given by $K \sim \sigma_{w}^{2} \tau$, where $\tau$ is the turbulence time scale (Holtslag and Moeng 1991). In the KPP scheme the diffusivity profile is specified through a similarity profile, using the velocity and length scales appropriate to the turbulent flow. Modifications to the KPP scheme to take account of Langmuir turbulence have been proposed (McWilliams and Sullivan 2000; Smyth et al. 2002) assuming that the velocity scale is $\sim u_{\mathrm{s} 0}$ (Smith 1998). However, without an understanding of the scaling of Langmuir turbulence, the accuracy of the proposed parameterizations remains unclear.

In this study, large-eddy simulation is used to investigate the TKE budget of Langmuir turbulence and based on this the appropriate velocity and length scales for Langmuir turbulence are proposed. The simulations are idealized and are not intended to be comprehensive simulations of turbulence in the oceanic mixed layer. This study focuses on mixing within the mixed layer and so the impact of wave breaking has not been considered. Observations show that the dissipation rate near the surface is significantly increased over the law of the wall in the presence of breaking waves (Agrawal et al. 1992; Terray et al. 1996). Recently Noh and Min (2004) and Sullivan et al. (2007) have included parameterizations of wave breaking in large-eddy simulations of Langmuir turbulence. Turbulence in the bulk of the mixed layer was not strongly affected by the presence of breaking waves, although both studies showed that the Langmuir circulations tend to become less coherent in the presence of wave breaking. An assumption of the present study is that a scaling for idealized Langmuir turbulence is relevant to the real ocean.

\section{Description of large-eddy simulations}

The Met Office large-eddy model (LEM) was used in this study, modified to represent the oceanic mixed layer. The model uses the Boussinesq approximation. Advection was done using the scheme described by Piacsek and Williams (1970) with a leapfrog time step, which conserves energy and scalar variances. The atmospheric version of the LEM is described in detail by Shutts and Gray (1994).

A number of changes were made to the LEM to simulate the oceanic mixed layer. An approximate equation of state (Bryden et al. 1999) was used to calculate the density, the thermal expansion, and saline contraction coefficients of seawater. These were used to calculate density fluctuations for the buoyancy term in the vertical velocity equation. The upper boundary condition was changed to a slip condition, using MoninObukhov similarity to diagnose the surface current from the imposed stress (Polton and Belcher 2007).

There is considerable uncertainty as to the appropriate value of the waterside roughness length. Craig and Banner (1994) suggested values ranging from 1 to $8 \mathrm{~m}$ based on comparisons between a closure model, with a representation of wave breaking and observed dissipation rates. Craig and Banner (1994) suggest that the larger 
roughness lengths obtained by fitting the model results to data probably reflected a failure of the model, citing the presence of Langmuir circulations as one possible cause. The general consensus is that the roughness length is much larger than the airside roughness length (Csanady 1997). For the present simulations the roughness length was taken to be $z_{0}=0.1 \mathrm{~m}$. Because the surface stress was imposed in these simulations the choice of roughness length only determines the surface current diagnostically, and the precise value should not be critical to the turbulence dynamics. To check this one of the simulations described was rerun using $z_{0}=0.5 \mathrm{~m}$. Differences in the turbulence from the original simulation were not significant.

The interaction of surface waves and currents are parameterized using the vortex force of Craik and Leibovich (1976), following Skyllingstad and Denbo (1995). The Stokes drift, $u_{s}$, was assumed to decrease exponentially with depth as $u_{s}=u_{\mathrm{s} 0} \exp (z / \delta)$, where $\delta$ is the Stokes depth scale, which is determined by the wavelength of the surface waves. The effects of subgrid turbulence were represented using a simple Smagorinskytype eddy viscosity (Smagorinsky 1963) modified as described by Brown et al. (1994).

The use of the Craik-Leibovich vortex force to parameterize the effects of Stokes drift involves phase averaging over the wave period, in addition to the spatial filtering of the LES model. It is not clear how, or whether, the subgrid model used in the LES should be modified to account for wave effects. McWilliams et al. (1997) considered that given the ad hoc basis for subgrid models it was difficult to see how they should be modified to account for wave effects. Since LES results are usually insensitive to the details of the subgrid model, and given the uncertainties, McWilliams et al. (1997) chose not to modify their subgrid model. In the present simulations, apart from the region close to the surface, subgrid contributions to turbulent fluxes are small suggesting that the details of the subgrid model are unlikely to be critical. It also worth noting that previous LES studies have used a variety of subgrid models and the characteristics of Langmuir turbulence from the present study are similar to those obtained in previous studies.

All simulations used $128 \times 128$ points in the horizontal, with a uniform grid spacing of $2 \mathrm{~m}$, and 111 points in the vertical with a uniform grid spacing of $0.8 \mathrm{~m}$. To prevent reflection of waves from the bottom boundary there was a damping layer below $65 \mathrm{~m}$ in which model fields were relaxed back to the horizontal mean. The time step was chosen to ensure stability of the model, and was varied as the run progressed. All simulations were run for $100000 \mathrm{~s}$, with statistics calculated over the last 40 000. Although there were inertial oscillations in the mean current, the turbulence fields were in a steady state over the averaging period.

The initial conditions from McWilliams et al. (1997) were used for all simulations. The initial mixed layer depth was $33 \mathrm{~m}$ with a uniform stratification of $0.01 \mathrm{~K} \mathrm{~m}^{-1}$ between $33 \mathrm{~m}$ and the bottom of the domain. The Coriolis parameter was taken to be $1 \times 10^{-4} \mathrm{~s}^{-1}$. Although the initial mixed layer depth was $33 \mathrm{~m}$, the final mixed layer depth, defined as the depth of the minimum in the buoyancy flux profile, varied between 34 and $42 \mathrm{~m}$. Unlike the simulation described by McWilliams et al. (1997) no surface buoyancy flux was imposed in the present simulations as there were no problems in spinning up turbulence from rest when wave effects were included.

The surface stress and the Stokes drift were varied so as to obtain a range of values for the turbulent Langmuir number, $\mathrm{La}_{t}$. The values of $\mathrm{La}_{t}$ were $0.15,0.2,0.3,0.4,0.8$, and 1.5. For each value of $\mathrm{La}_{t}$ three simulations were carried out with $u_{*}=0.0043,0.0060$, and $0.0085 \mathrm{~m} \mathrm{~s}^{-1}$. For most simulations the Stokes depth was set to $4.8 \mathrm{~m}$. To test the sensitivity of the results to the value of $\delta$ three simulations with $\mathrm{La}_{t}=0.3$ and $u_{*}=0.006 \mathrm{~m} \mathrm{~s}^{-1}$ and $\delta=3.1,6.4$, and $9.5 \mathrm{~m}$ were also run. Smaller values of $\delta$ were not considered because of resolution.

One simulation without the Stokes forcing was also carried for comparison. This had $u_{*}=0.0085 \mathrm{~m} \mathrm{~s}^{-1}$ and $f=$ $1 \times 10^{-4} \mathrm{~s}^{-1}$. For this simulation a small surface buoyancy flux, equivalent to a sensible heat flux of $5 \mathrm{~W} \mathrm{~m}^{-2}$, was imposed to ensure that turbulence developed. The Obukhov length for this simulation is, $L=-148 \mathrm{~m}$, and the mixed layer depth, $h$, was $34 \mathrm{~m}$, so $h / L=-0.24$, indicating that buoyancy effects are not significant (Holtslag and Nieuwstadt 1986).

\section{The turbulent kinetic energy budget}

For stationary, horizontally homogeneous conditions, the TKE budget including wave effects can be written as (Kitaigorodskii and Lumley 1983; Polton and Belcher 2007)

$$
\begin{gathered}
-\overline{u^{\prime} w^{\prime}} \frac{\partial \bar{U}}{\partial z}-\overline{v^{\prime} w^{\prime}} \frac{\partial \bar{V}}{\partial z}-\overline{u^{\prime} w^{\prime}} \frac{d u_{s}}{d z}+\overline{w^{\prime} b^{\prime}} \\
-\frac{\partial}{\partial z}\left(\overline{w^{\prime} E}+\frac{1}{\rho} \overline{w^{\prime} p^{\prime}}\right)-\epsilon=0,
\end{gathered}
$$

where $\bar{U}$ and $\bar{V}$ are the horizontal components of the current parallel and perpendicular, respectively, to the surface stress; the overbar denotes an average; $w$ is the vertical component of the current, taken to be positive upward; $\rho \overline{u^{\prime} w^{\prime}}$ and $\rho \overline{v^{\prime} w^{\prime}}$ are the components of Reynolds stress vector, where the primes denote fluctuations from the mean; $\overline{w^{\prime} b^{\prime}}$ is the turbulent buoyancy 

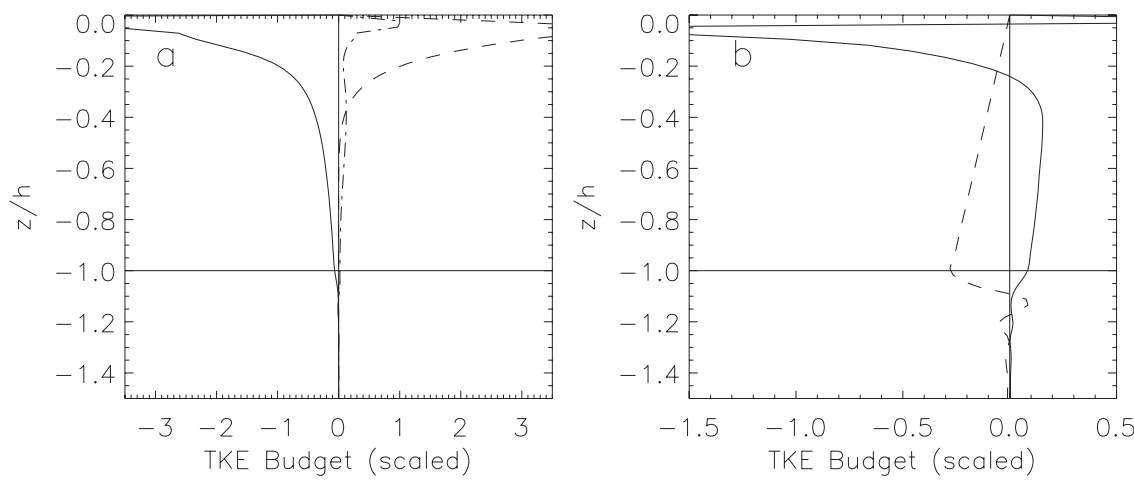

FIG. 1. Example of the TKE budget for $\mathrm{La}_{t}=0.3$. The terms have been scaled by $w^{3} *_{L} / h$ (see text). (left) Dissipation rate (full curve), Stokes production (dashed curve), shear production (dash-dot curve). (right) Transport (full curve) and buoyancy flux (dashed curve). The buoyancy flux has been multiplied by a factor of 5 for clarity.

flux; $\overline{w^{\prime} E}$ is the turbulent energy flux; $\overline{w^{\prime} p^{\prime}}$ is the pressure work flux; $\epsilon$ is the dissipation rate due to molecular viscosity; and $\rho$ is density. The Stokes drift is assumed to be parallel to the surface stress.

In Eq. (1) the first two terms on the left-hand side represent production of TKE due to shear in the mean current, the third term is production due to the Stokes shear, the fourth is the production or destruction of TKE through buoyancy forces, the fifth term represents transport of TKE due to turbulent fluctuations of the vertical velocity and pressure, and the final term is the destruction of TKE due to viscosity. The three shear terms in Eq. (1) feed energy into different velocity components. The terms involving the current shear feed energy into the horizontal turbulent velocities, while the term involving the Stokes shear feeds energy into the vertical component. Note that the Stokes term does not feed energy directly into the lateral component of the current; rather there is an exchange of energy between the lateral and vertical components, which does not appear in the TKE budget (Skyllingstad and Denbo 1995).

In the results to be presented the terms in the TKE budget were calculated directly from the discretized LES equations so as to be consistent with the grid staggering and these terms, calculated at each time step, were averaged over space and time, rather than using the average stress and mean current shears as suggested in Eq. (1). Calculating the terms directly from the LES equations gives a budget residual that is less than $0.5 \%$ of the dissipation rate. Calculating the shear terms from the fluxes and shears gives a residual of order $10 \%$, mainly due to the Stokes term, although qualitatively the results are similar to the directly calculated terms. Note that because the time stepping and advection schemes conserve energy all of the dissipation is associated with the subgrid model (Brown et al. 2000).
Figure 1 shows an example of a TKE budget of the resolved motions from one simulation [this simulation has the same parameters as described by McWilliams et al. (1997)]. The shear production terms and dissipation are shown in Fig. 1a and the buoyancy and transport terms are shown in Fig. 1b. The budget is similar to those obtained by McWilliams et al. (1997) and Polton and Belcher (2007). The production of TKE is dominated by the Stokes term above $z / h \approx-0.3$, with the production of TKE through the mean current shear being small. However, unlike the production through Stokes shear, the production of TKE through current shear extends below $z / h=-0.3$, where, for this simulation, it is about $20 \%$ of the dissipation rate. The dissipation rate decreases rapidly with depth close to the surface and more gradually below $z / h=-0.3$.

The difference between production through current shear and dissipation below $z / h=-0.3$ is balanced by the transport term, which is shown in Fig. 1b. Turbulent kinetic energy is transported from the region near the surface in which production of TKE by the Stokes shear exceeds dissipation, to the rest of the mixed layer, where it acts as a source of TKE. The buoyancy term is comparable to the transport term in magnitude and, by definition, is a minimum at the base of the mixed layer. The buoyancy term is a sink of TKE, and is associated with the entrainment of cold water into the mixed layer.

Figure 2a shows the components of the transport term from the simulation shown in Fig. 1. The turbulent transport term been split into two components: one associated with the flux of TKE in the horizontal velocity components [i.e., $\left.1 / 2\left(\overline{w^{\prime} u^{\prime 2}}+\overline{w^{\prime} v^{\prime 2}}\right)\right]$ and the other with the flux of TKE in the vertical velocity component, $\overline{w^{\prime 3}} / 2$. The turbulent transport term is dominated by the transport of $\overline{w^{\prime 2}}$, the flux of TKE associated with the horizontal velocity components being much smaller. 

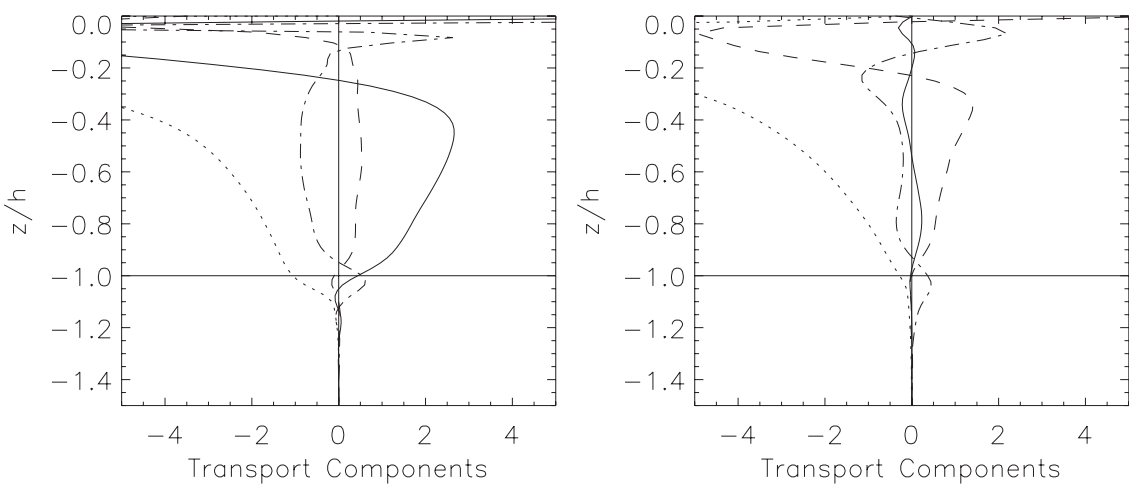

FIG. 2. (left) Components of the transport term: $(-1 / 2) \partial \overline{w^{\prime 3}} / \partial z$ (solid curve), $-1 / 2 \partial \overline{\left(w^{\prime} u^{\prime 2}\right.}+$ $\left.\overline{w^{\prime} v^{\prime 2}}\right) / \partial z$, (dashed curve) $-1 / \rho \partial \overline{w^{\prime} p^{\prime}} / \partial z$ (dash-dot curve), and dissipation rate (dotted curve). All quantities have been scaled by $u_{*}^{3} / h$. (right) As in (left), but for a shear-only simulation.

Figure $2 \mathrm{~b}$ shows the components of the transport term for the shear-only simulation. In contrast to the simulation with wave effects, in this simulation the transport of horizontal TKE is larger than that associated with the vertical component of TKE. This is consistent with the simulations of the neutral atmospheric boundary layer described by Moeng and Sullivan (1991). Overall the transport of TKE, nondimensionalised by $u_{*}^{3} / h$ is smaller in the simulation without the wave effects. In both simulations the pressure term is opposite in sign and smaller than the turbulent transport. This is qualitatively similar to results from simulations of both the shear-driven and convective atmospheric boundary layer (Moeng and Sullivan 1991).

The difference in the components of the turbulent transport term reflects the difference between Stokes production and production of TKE through current shear. The Stokes term acts as a source of TKE in the vertical component of the current, while the current shear is a source of horizontal TKE. Polton and Belcher (2007) identified downwelling jets that originated in the region of Stokes shear and penetrated deep into the mixed layer. The results in Fig. 2 indicate that these downwelling jets are responsible for the turbulent transport of TKE from the region of Stokes shear into the bulk of the mixed layer.

\section{Scaling Langmuir turbulence}

Outside of the surface layer the scaling parameters for shear-driven turbulence are the surface friction velocity, $u_{*}$, and the mixed layer depth, $h$, so that quantities made nondimensional by $u *$ and $h$ should be functions of $z / h$ alone. Including wave effects, as parameterized by the Craik-Leibovich vortex force, there are additional dimensional parameters: the surface Stokes drift, $u_{s 0}$, and the Stokes depth, $\delta$. Two nondimensional pa- rameters can be formed: the turbulent Langmuir number, $\operatorname{La}_{t}^{2}=\left(u_{*} / u_{s 0}\right)$ and $\delta / h$. Li et al. (2005) considered the variation of the nondimensional variance of the vertical component of the current, $\sigma_{w}^{2} / u_{*}^{2}$ with $\mathrm{La}_{t}$, while more recently Harcourt and D'Asaro (2008) have considered the dependence on $\delta / h$. The choice of $u *$ for the velocity scale for the shear-driven mixed layer reflects the production of TKE from the current shear terms in the TKE budget. Figure 1 shows that with wave effects present the current shear term may be very small, and so the friction velocity alone no longer reflects the process generating TKE. We can ask whether a scale can be developed for turbulence in which the main production term is associated with the Stokes shear?

For high Reynolds number turbulence the dissipation rate is determined by the characteristics of the large eddies (Tennekes and Lumley 1972). If the characteristic velocity of the large eddies in the turbulent flow is $\nu *$ and their length scale is $\lambda$, the magnitude of the dissipation rate is $\nu_{*}^{3} / \lambda$. For Langmuir turbulence a velocity scale, $w_{* L}$, can be defined through the relation $\epsilon \sim w_{*}^{3}{ }^{3} / \lambda$ by assuming that the dissipation rate balances the dominant production term in the TKE budget (i.e., the Stokes production; Fig. 1).

The region of significant production of TKE through the Stokes shear is quite shallow $[O(\delta)]$, but because of the transport of TKE from this layer dissipation of TKE occurs over the full depth of the mixed layer. This suggests that the relevant length scale for the large eddies is the mixed layer depth, $h$, rather than the Stokes depth scale, $\delta$. Simulations described by Polton and Belcher (2007) did not include stratification and in that case turbulence extended to a depth of $\approx u * / f$. In the present simulations, $f h / u_{*}$ is always less than 1 (with values between 0.5 and 0.8 ) and so stratification limits the depth of mixing, but for deeper pycnoclines $u_{*} / f$ would be the relevant length scale. 
The production rate of TKE in the layer of significant Stokes shear can be estimated as $u_{*}^{2} u_{\mathrm{s} 0} / \delta$ and the average production over the mixed is $u_{*}^{2} u_{\mathrm{s} 0} / \delta(\delta / h) \sim u_{*}^{2} u_{\mathrm{s} 0} / h$. Equating this estimate for the production term to dissipation gives

$$
w_{*_{L}}=\left(u_{*}^{2} u_{\mathrm{s} 0}\right)^{1 / 3} .
$$

Smith (1996) suggested Eq. (2) as the velocity scale for the transverse velocity of the circulations close to the surface, $\sigma_{v}$, although it is not clear from his derivation if it is relevant to other quantities.

The scale $w_{* L}$ can also be written in terms of $u *$ and $\mathrm{La}_{t}$ as

$$
w_{*_{L}}=\frac{1}{\mathrm{La}_{t}^{2 / 3}} u *
$$

The turbulent Langmuir number (or strictly $\mathrm{La}_{t}^{2}$ ) can be interpreted as the ratio of the production of TKE by the current shear $\left(\sim u_{*}^{3} / h\right)$ to the production due to Stokes shear $\left(\sim u_{*}^{2} u_{\mathrm{s} 0} / h\right)$. For large $\mathrm{La}_{t}$ production of TKE due to the current shear dominates and the relevant turbulent velocity scale is $u_{*}$, while for small $\mathrm{La}_{t}$ the Stokes production dominates and $w_{* L}$ is the relevant velocity scale. With this interpretation of $\mathrm{La}_{t}$ it is clear that if the proposed scaling is correct as $\mathrm{La}_{t}$ becomes small, turbulent properties made nondimensional using $w *{ }_{, L}$ should not be functions of $\mathrm{La}_{t}$.

Harcourt and D'Asaro (2008) have recently suggested Eq. (3) based on an analysis of $\sigma_{w}^{2} / u_{*}^{2}$ obtained from a series of large-eddy simulations. They found that $\sigma_{w}^{2} / u_{*}^{2} \propto \mathrm{La}_{t}^{-4 / 3}$, which is consistent with Eq. (2) if $\sigma_{w}^{2} / w_{* L}^{2}=$ constant, which should be the case if $w_{* L}$ is the relevant velocity scale. Harcourt and D'Asaro (2008) pointed out that this result is consistent with a balance between Stokes production and dissipation for eddies with scales of the order of the mixed layer depth, based on the behavior of $\sigma_{w}^{2} / u_{*}^{2}$ as $\delta / h \rightarrow 0$.

\section{The nondimensional TKE budget}

\section{a. Turbulent dissipation: Simulations}

The simulations considered in this section all have the same Stokes depth scale, $\delta=4.8 \mathrm{~m}$. The effects of variations in $\delta$ will be considered later.

Figure 3 shows the dissipation profiles from the LES simulations scaled by $w_{* L}^{3} / h$. As $\mathrm{La}_{t}$ decreases, the magnitude of $\epsilon h / w_{* L}^{3}$ decreases until $\mathrm{La}_{t} \approx 0.5$. For $\mathrm{La}_{t}<0.5$ the dissipation profiles collapse to a single curve, with the variation in the scaled profiles being much smaller than the variation in the dimensional profiles. This is indicated in the figure by the range in the scale $w *_{L}^{3} / h$ for the

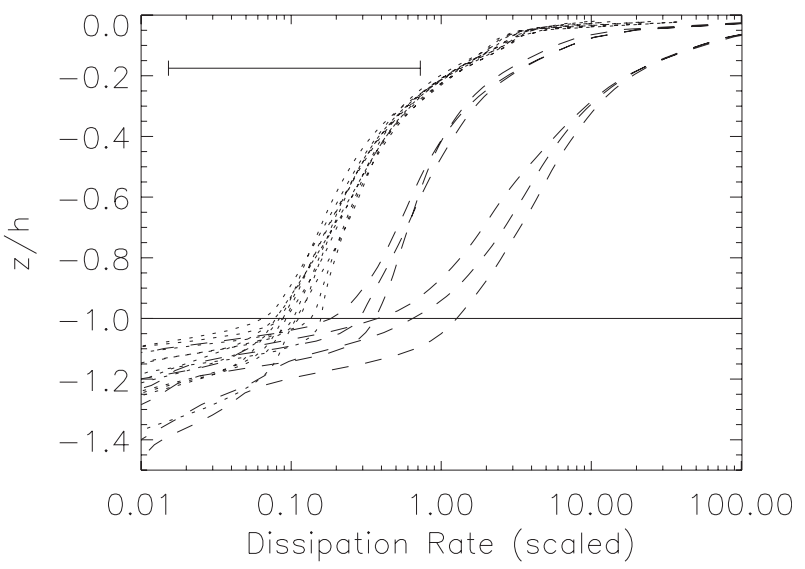

FIG. 3. Dissipation rates scaled by $w_{* L}^{3} / h$ as a function of $z / h$. Simulations with $\mathrm{La}_{t}<0.5$ (dotted curves). Dissipation rates for simulations with $\mathrm{La}_{t}=0.8$ and $\mathrm{La}_{t}=1.5, \mathrm{La}_{t}$ increasing from left to right (dashed curves). The horizontal line at $z / h=-0.2$ shows the variation of $w_{*}^{3} / h$ among the simulations with $\mathrm{La}_{t}<0.5$.

simulations with $\mathrm{La}_{t}<0.5$. The relative variation in the scaled profiles increases with depth. The variation in dissipation rate around the base of the mixed layer will be considered in more detail in section 7.

The dissipation rate at $z / h=-0.5$, scaled by $u_{*}^{3} / h$, is shown in Fig. 4 as a function of $\mathrm{La}_{t}$. For small values of $\mathrm{La}_{t}$, for which the Stokes production dominates, Eq. (3) implies that $\epsilon h / u_{*}^{3} \propto 1 / \mathrm{La}_{t}^{2}$. For $\mathrm{La}_{t}<0.5$ the dissipation rate varies as $1 / \mathrm{La}_{t}^{2}$, consistent with the results in Fig. 3 . For $\mathrm{La}_{t}>0.5$ the dissipation rate in the middle of the mixed layer is less than for shear turbulence, even for $\mathrm{La}_{t}=2$ (note, the shear-only simulation has been assigned $\mathrm{La}_{t}=4$ for display purposes). This variation in dissipation rate with $\mathrm{La}_{t}$ means that the nondimensional dissipation for $\mathrm{La}_{t} \approx 0.3$ is similar in magnitude to the dissipation rate for shear-driven turbulence, without wave effects.

The variation in dissipation rate with Langmuir number for $\mathrm{La}_{t}>0.5$ is due to the way in which production of TKE by Stokes shear and current shear varies as $\mathrm{La}_{t}$ increases. With increasing $\mathrm{La}_{t}$ the Stokes production, nondimensionalised by $u_{*}^{3} / h$, decreases while production of TKE by current shear increases. However, initially the decrease in the Stokes production with increasing $\mathrm{La}_{t}$ is greater than the increase in production through current shear, which leads to an overall decrease in the production of TKE and hence a decrease in the dissipation rate. As $\mathrm{La}_{t}$ continues to increase the current shear production increases and the wave effects become negligible.

The results in Figs. 3 and 4 show that there is a distinct Langmuir turbulence regime that occurs for $\mathrm{La}_{t}<0.5$ and is characterized by the velocity scale, $w_{*_{L}}$. 


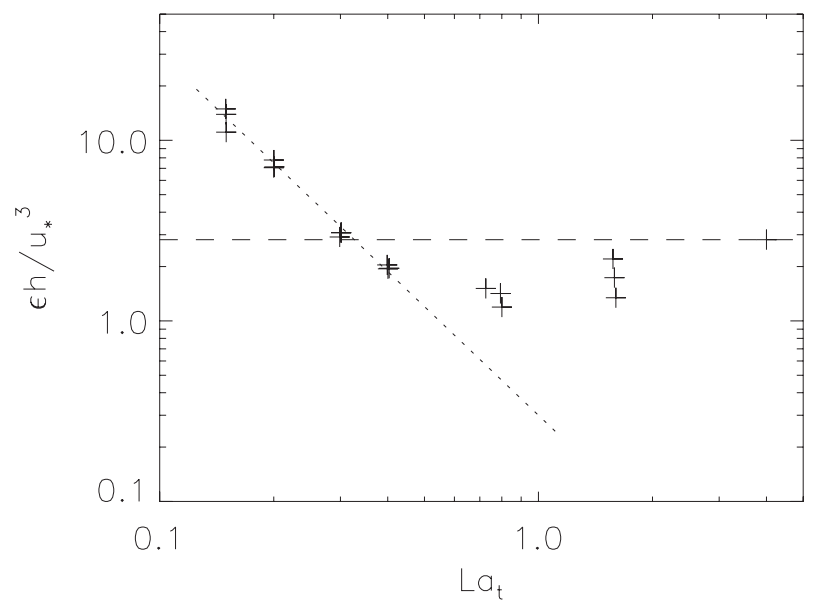

FIG. 4. Nondimensional dissipation rate, $\epsilon h / u_{*}^{3}$ as a function of the turbulent Langmuir number. Here $\epsilon h / u_{*}^{3}=0.3 / \mathrm{La}_{t}^{2}$ (dotted line). The point at $\mathrm{La}_{t}=4$ is for the simulation without wave effects.

\section{b. Turbulent dissipation: Observations}

Estimates of the dissipation rate in the oceanic mixed layer are reasonably common, but information on the wave field needed to determine the Stokes drift, and hence $\mathrm{La}_{t}$, are rarely given. This means that it is not possible to make a direct comparison between the LES scaling for Langmuir turbulence derived here with observations. The best that can be done is to compare the shapes of observed dissipation profiles with those from the LES. Figure 5a compares the model profiles obtained from the present simulations with $\mathrm{La}_{t}<0.5$ with observed dissipation profiles published by Greenan et al. (2001), Thorpe et al. (2003), and Lozovatsky et al. (2006), all for conditions when surface buoyancy effects in the mixed layer were small. Langmuir circulations were observed to be present by Thorpe et al. (2003). The observed profiles have been multiplied by a factor (different for different sets of observations) to match the magnitude of the model profiles so as to allow the shapes of the profiles to be compared. Based on Eq. (3) the Langmuir numbers for these profiles are in the range of $0.15-0.30$. On average the effective $\mathrm{La}_{t}$ for these data is smaller than expected for fully developed seas. Greenan et al. (2001) presented wave spectra that showed two peaks due to swell in addition to peak for the wind-driven waves, which may explain the low value of $\mathrm{La}_{t}(=0.15)$ implied by their data.

The shapes of the LES dissipation profiles agree well with the observations. The dissipation rate decreases rapidly down to $z / h=-0.3$, and less rapidly below this. The observations from Thorpe et al. (2003) capture the region just below the surface and the upper half of the mixed layer, while the data from Greenan et al. (2001) and Lozovatsky et al. (2006) show the more gradual

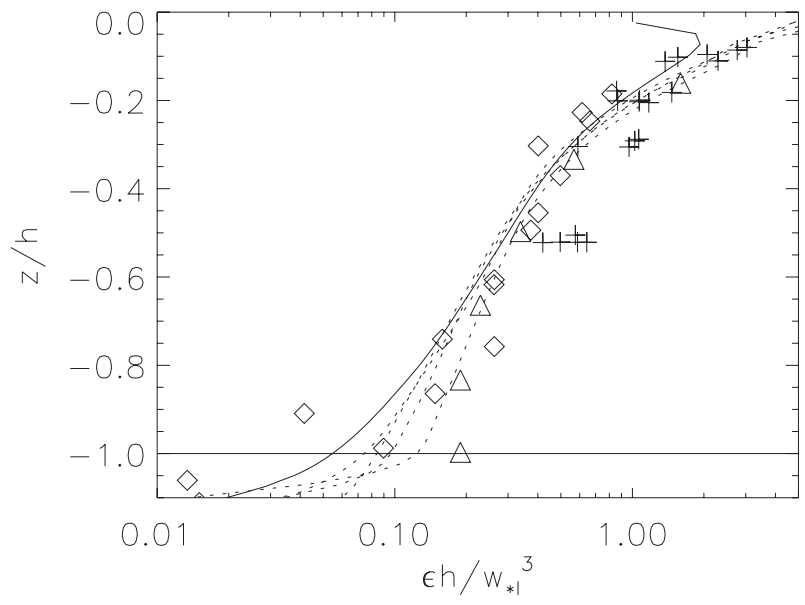

FIG. 5. Comparison of the shapes of the nondimensional dissipation rate, $\epsilon h / w^{3}{ }_{L}$, from the LES with adjusted observations. The results from the LES for $\mathrm{La}_{t}<0.5$ (dotted curves), observed profiles (symbols), with magnitudes adjusted to coincide with the simulations (see the text). From Lozovatsky et al. (2006) (diamonds), from Greenan et al. (2001) (triangles), and from Thorpe et al. (2003) (plus signs). The magnitudes of the observed dissipation rates have been adjusted to match the LES profiles. The dissipation from the simulation of shear-driven turbulence (solid curve).

decrease over the rest of the mixed layer. Also shown in Fig. 5 is the dissipation rate obtained from the simulation without wave effects. The shape of this profile is similar to those for Langmuir turbulence, although the decrease in the dissipation rate over the depth of the mixed layer appears to be slightly greater than for Langmuir turbulence. While this difference may not be significant, since the profile for shear-driven turbulence falls just outside of the variation in the dissipation profiles shown in Fig. 3 for $\mathrm{La}_{t}<0.5$, it could reflect the smaller magnitude of the transport of TKE in the shear turbulence simulations compared to that in the simulations of Langmuir turbulence. Figure 5 suggests that the shape of the dissipation profile is not diagnostic of Langmuir or shear-driven turbulence.

The dissipation length scale, $\lambda_{\epsilon}$ is defined as

$$
\lambda_{\epsilon}=\frac{E^{3 / 2}}{\epsilon}
$$

(Tennekes and Lumley 1972) and is characteristic of the length scale of the energy containing eddies of a turbulent flow. Figure 6 a shows the dissipation length scale for the present simulations with $\mathrm{La}_{t}<0.5$, and for the shear-only simulation. From the surface to about $z / h=$ -0.5 the dissipation length scale from the simulations of Langmuir turbulence is proportional to depth. In the lower half of the mixed layer $\lambda_{\epsilon}$ is approximately equal to the mixed layer depth. The shear-only simulation differs from the Langmuir turbulence results in 


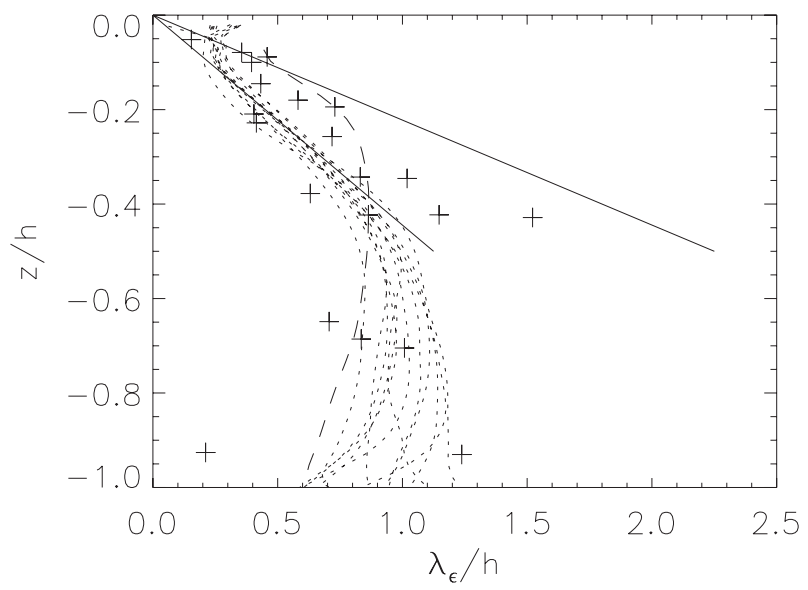

FIG. 6. The dissipation length scale $E^{3 / 2} / \epsilon$, normalized by mixed layer depth as a function of nondimensional depth. Simulations with $\mathrm{La}_{t}<0.5$ (dotted curves), simulation with no wave effects (dashed curve), $\lambda_{\epsilon}=2 z$ and $\lambda_{\epsilon}=4.5 z$ (solid curves). Observations from the neutral atmospheric boundary layer (symbols).

that the increase with depth below the surface is more rapid and $\lambda_{\epsilon}$ is approximately constant over a larger fraction of the mixed layer depth. The general similarity between the dissipation length scale for Langmuir and shear turbulence is due to the size of the turbulent eddies being limited either by distance from the surface or the presence of the thermocline, irrespective of the production mechanism.

Estimates of the dissipation length scale in the neutral atmospheric boundary layer (Grant 1992) are also in- cluded in Fig. 6. The observed profile of the dissipation length scale in the neutral atmospheric boundary layer and that derived from the Stokes-forced LES are similar. Unfortunately the scatter in the data is too large to distinguish between shear and Langmuir turbulence. The dissipation length scale confirms that the mixed layer depth is the relevant length scale for Langmuir turbulence when $\delta / h$ is small.

\section{c. Shear production}

Figures 7a-c show the scaled Stokes production (Fig. 7a), total shear production (Stokes + current; Fig. 7b), and the transport term in the TKE budget (Fig. 7c) for simulations with $\mathrm{La}_{t}<0.5$. For comparison the scaled dissipation rate, plotted on a linear scale is shown in Fig. 7d. As in the example shown in Fig. 1, TKE production due to Stokes shear is the dominant source of TKE, although in some of the simulations there are regions in which the Stokes production is negative (i.e., it is a sink of TKE). Although generally small, comparison between the Stokes production and total shear production (Figs. 7a,b) shows that the TKE production associated with mean current shear is not unimportant. Unlike the Stokes production the total shear production is always positive, and collapses more nearly on to a single similarity curve than is the case for the Stokes production alone. Although not perfect, the near collapse of the shear production term shows that it scales with $w_{*}^{3} / h$ rather than $u_{*}^{3} / h$. When scaled with $u_{*}^{3} / h$, there is much more variation due to variations in $\mathrm{La}_{t}$ (not shown). The
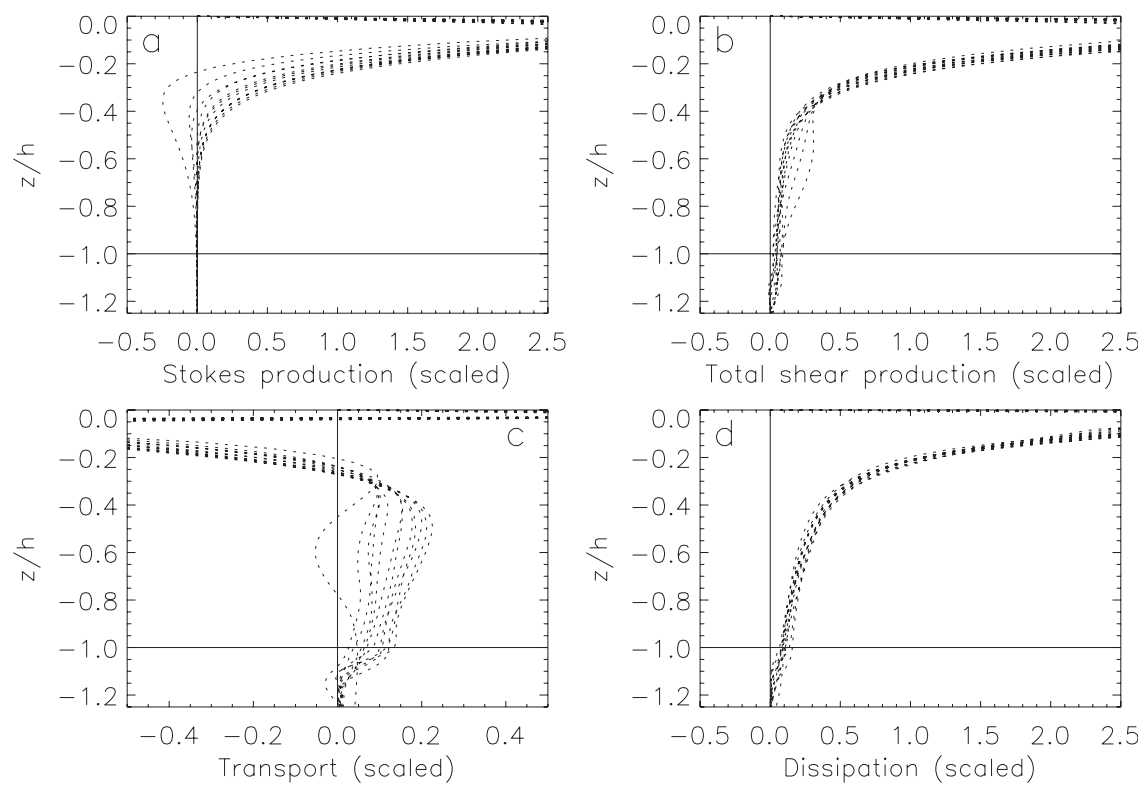

FIG. 7. Terms in the TKE budget normalized by $w^{3} *_{L} / h$. Results are for simulations with $\mathrm{La}_{t}<0.5$. (a) Stokes production, (b) total shear production, (c) transport (note the different scale), and (d) dissipation rate. 

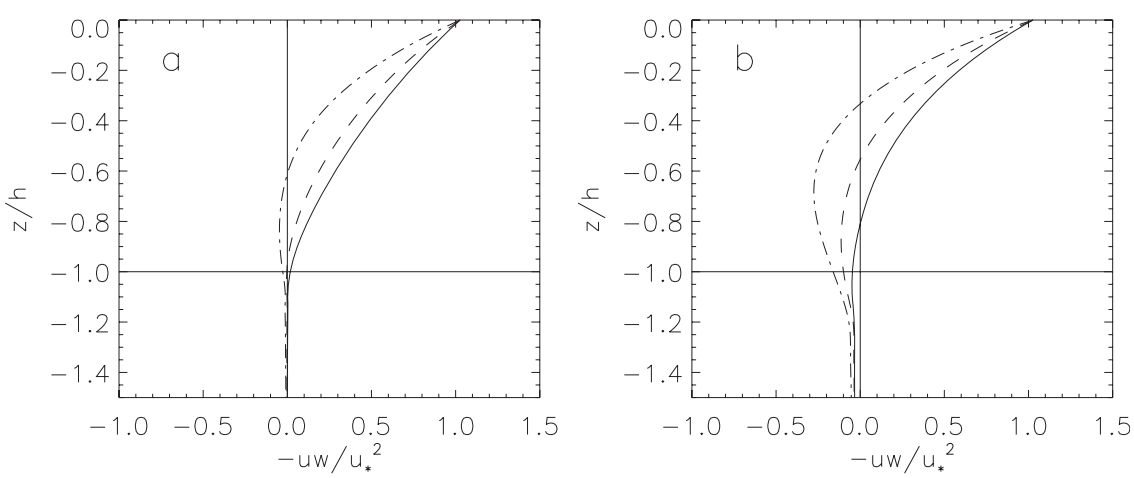

FIG. 8. Profiles of the stress component parallel to the surface stress. (a) Profiles from simulations with $\mathrm{La}_{t}=0.3$. The three values of the parameter $f h / u_{*}$ (three curves), $f h / u_{*}=0.46$ (solid curve), $f h / u *=0.62$ (dashed curve), and $f h / u *=0.85$ (dash-dot curve). (b) As in (a), but for simulations with $\mathrm{La}_{t}=0.2$.

scaling by $w_{*}^{3} L / h$ reflects the effect of mixing by Langmuir turbulence on the mean shear.

In common with previous LES studies the CoriolisStokes forcing has been included in the present LES. Polton et al. (2005) used an analytic model to investigate Coriolis-Stokes forcing in the oceanic mixed layer and concluded that observed current profiles agreed better with modeled profiles that included CoriolisStokes forcing than results for a simple Ekman layer. The presence of this Coriolis-Stokes forcing means that the Stokes drift affects the stress profiles through the mean momentum budget, and hence the Stokes-shear production in the TKE budget.

Figures $8 \mathrm{a}, \mathrm{b}$ shows examples of profiles of $\overline{u^{\prime} w^{\prime}}$ for three values of $u *$ from simulations with $\mathrm{La}_{t}=0.3$ (Fig. 8a) and $\mathrm{La}_{t}=0.2$ (Fig. 8b). The profiles are generally curved, with the curvature being larger for the smaller values of Langmuir number. For a given value of $\mathrm{La}_{t}$ the curvature of the $\overline{u^{\prime} w^{\prime}}$ profiles increases with decreasing $u *$. For $\mathrm{La}_{t}=0.2$ the $\overline{u^{\prime} w^{\prime}}$ changes sign in some of the simulations, and it is this that is responsible for the negative Stokes production.

These variations in the shape of the stress profiles can be understood from mean momentum budget that for horizontally homogeneous conditions, can be written as

$$
\frac{1}{\rho} \frac{\partial \boldsymbol{\tau}}{\partial z}=-\frac{\partial \overline{\mathbf{U}}}{\partial t}+f \mathbf{k} \times\left(\overline{\mathbf{U}}+\mathbf{u}_{s}\right)
$$

(e.g., Polton et al. 2005). Taking the stress gradient to be $\sim \rho u_{*}^{2} / h$, and the Stokes drift as $\sim u_{s 0}$, Eq. (5) can be written in nondimensional form as

$$
\frac{1}{\rho} \frac{\partial \widetilde{\tau}}{\partial z}=-\frac{h}{u_{*}} \frac{\partial \widetilde{\mathbf{U}}}{\partial t}+\frac{f h}{u_{*}} \mathbf{k} \times\left(\widetilde{\widetilde{\mathbf{U}}}+\frac{1}{\mathrm{La}_{t}^{2}} \tilde{\mathbf{u}}_{\mathrm{s}}\right),
$$

where $\tilde{\overline{\mathbf{U}}}=\overline{\mathbf{U}} / u_{*}$ and $\tilde{u}_{s}=u_{s} / u_{\mathrm{s} 0}$ are nondimensional mean current and Stokes drift. As $f h / u_{*} \rightarrow 0$ there is no steady solution for Eq. (5). In this case the mixed layer current should accelerate uniformly with depth, and the stress profiles will be unidirectional, varying linearly with depth. For finite values of $f h / u_{*}$ the presence of Stokes drift in Eq. (6) means that the nondimensional stress gradient close to the surface should depend on $(f h / u *) \mathrm{La}_{t}^{-2}$, This argument suggests that for a given value of $f h / u_{*}$ the curvature of the stress profiles should increase as $\mathrm{La}_{t}$ decreases, which is consistent with Figs. 8a,b. For a fixed value of $\mathrm{La}_{t}$, the stress profile should become more curved as $f h / u *$ increases (e.g., because of $u_{*}$ decreasing), again consistent with Figs. 8a,b.

Figure 9a shows $-\left(h / u_{*}^{2}\right) \partial \overline{u^{\prime} w^{\prime}} / \partial Z$ at the surface as a function of $\left(f h / u_{*}\right) \mathrm{La}_{t}^{-2}$. As expected the nondimensional gradient of $\overline{u^{\prime} w^{\prime}}$ increases in magnitude with increasing $f h / u_{*} \mathrm{La}_{t}^{-2}$, and tends to 1 as $f h / u * \mathrm{La}_{t}^{-2} \rightarrow 0$. Figure $9 \mathrm{~b}$ shows the same for $-\partial \overline{v^{\prime} w^{\prime}} / \partial z$. In this case the gradient tends to 0 as $f h / u * \mathrm{La}_{t}^{-2} \rightarrow 0$, and the stress profile becomes unidirectional. This variation in the stress gradient near the surface affects the magnitude of the shear stress in the region of Stokes shear, and hence the Stokes-shear production in the TKE budget, as shown in Fig. 7a.

\section{d. The effect of the Stokes depth scale on TKE budget}

The simulations considered so far have had the same value for the Stokes depth scale $\delta$. Figures 10a-d show the terms in the TKE budget for simulations in which the Stokes depth scale is varied, but $u_{*}$ and $u_{s 0}$ are kept the same. As the Stokes depth scale increases the Stokes production extends to greater depths. The production of TKE associated with the mean current shear is small for these simulations, although below $z / h=-0.6$, it is responsible for about $25 \%$ of the production TKE. The dissipation profile shows less variation than the shear 

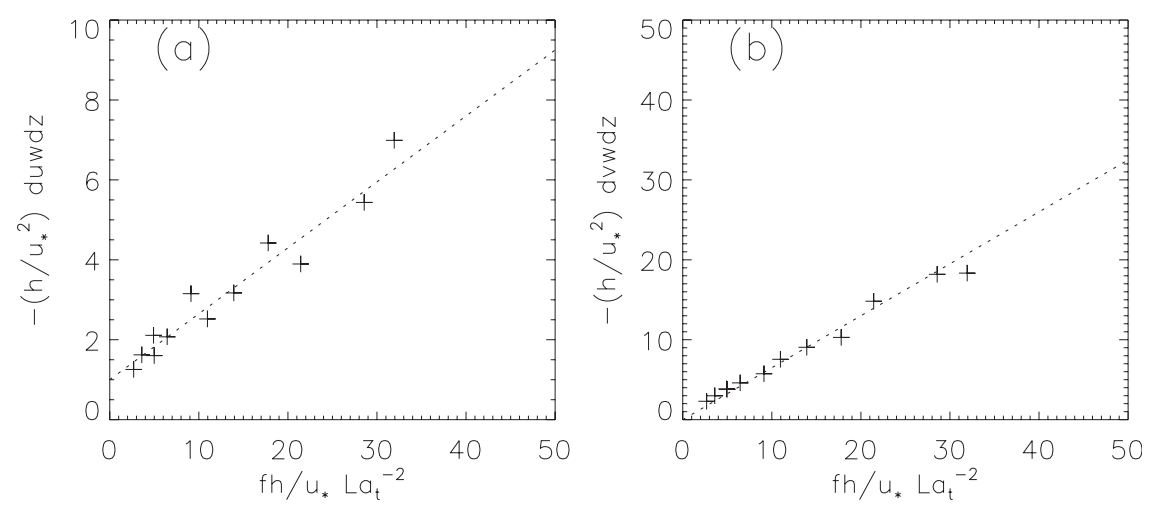

FIG. 9. Variation of the nondimensional stress gradient at the surface with $f h / u_{*} \mathrm{La}_{t}^{-2}$. (a) Stress component parallel to the imposed surface stress. (b) Component perpendicular to the imposed surface stress.

production profiles. The insensitivity of the dissipation profile to changes in the Stokes depth scale reflects changes in the transport term that are correlated with the changes in the total shear production. The changes in the transport term in the TKE budget with variations in the Stokes depth are consistent with the physical picture of Langmuir turbulence suggested by Polton and Belcher (2007).

\section{Turbulence statistics}

\section{a. Velocity variances}

Figure 11a shows an example of the profiles of the variances of the three velocity components. The profiles are similar to those obtained in previous LES studies (Skyllingstad and Denbo 1995; McWilliams et al. 1997; Polton and Belcher 2007). Over a large part of the mixed layer the variance of vertical velocity, $\sigma_{w}^{2}$, is larger than the variances of other components, while the variance of the velocity component parallel to the surface stress is the smallest. This is the opposite of what is seen in shear turbulence, and is a consequence of the Stokes production term feeding energy into the vertical velocity component, while the current shear production term feeds energy into the horizontal components. Linear vorticity dynamics in rapid distortion theory captures these differences (Teixeira and Belcher 2002).
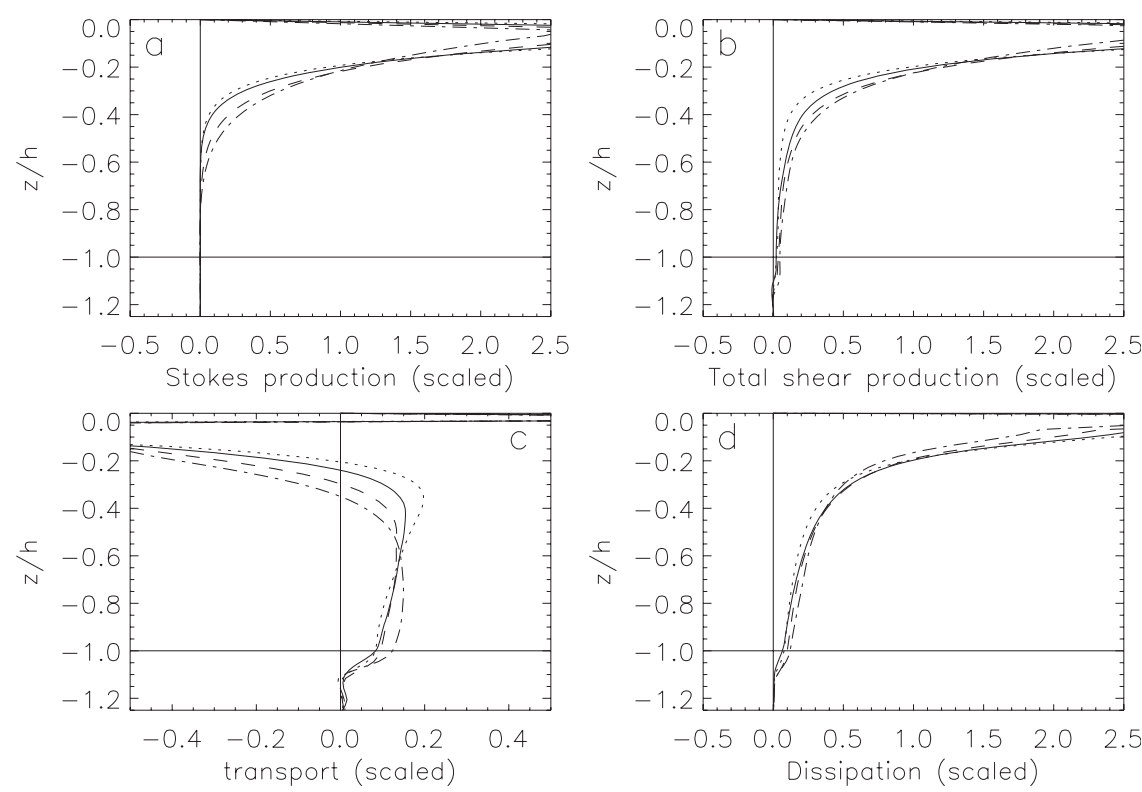

FIG. 10. Sensitivity of the TKE budget to variations in the Stokes depth scale, $\delta$. All simulations have $\mathrm{La}_{t}=0.3$. (a) Stokes production (dotted curve) $\delta=3.2 \mathrm{~m}, \delta=4.8 \mathrm{~m}$ (solid curve), $\delta=6.4 \mathrm{~m}$ (dashed curve), and $\delta=9.5 \mathrm{~m}$ (dash-dot curve). (b) Total shear production. (c) As in (a), but for transport. (d) As in (a), but for dissipation. 

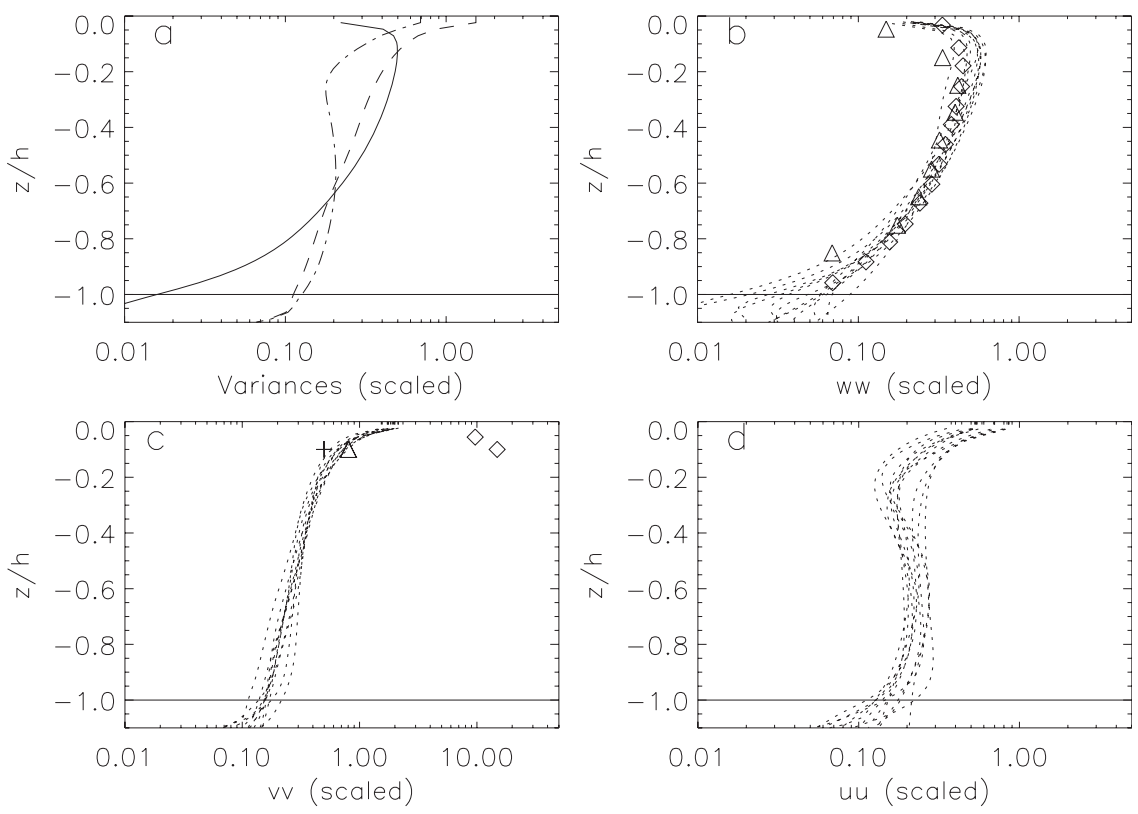

FIG. 11. (a) Example of variance profiles for individual velocity components $\sigma_{w}^{2}$ (full curve), $\sigma_{v}^{2}$ (dashed curve), and $\sigma_{u}^{2}$ (dash-dot curve). (b) Profiles of $\sigma_{w}^{2}$ scaled by $w_{*_{L}}^{2}$. The symbols are from D'Asaro (2001) (diamonds) and Tseng and D'Asaro (2004) (triangles). For the observed profiles a value of $\mathrm{La}_{t}=0.3$ has been assumed. (c) As in (a), but for $\sigma_{v}^{2}$. The symbols are observations from Plueddemann et al. (1996) (diamonds) and Smith (1998) (cross and triangle). (d) As in (a), but for $\sigma_{u}^{2}$

Toward the base of the mixed layer, and close to the surface, $\sigma_{w}^{2}$ is smaller then either of the other variances as a consequence of vertical motions being inhibited near interfaces and energy being distributed into the horizontal components (Hunt and Graham 1978).

Figures $11 \mathrm{~b}-\mathrm{d}$ show $\sigma_{w}^{2} / w_{*_{L}}^{2}, \sigma_{v}^{2} / w_{*_{L}}^{2}$ and $\sigma_{u}^{2} / w_{*_{L}}^{2}$ for simulations with $\mathrm{La}_{t}<0.5$. An idea of how well the scaling works can be gained by noting that there is a factor of 10 variation in $w_{* L}^{2}$ for the simulations shown in Fig. 11. D'Asaro (2001) and Tseng and D'Asaro (2004) present profiles of $\sigma_{w}^{2} / u_{*}^{2}$ obtained from Lagrangian floats. Since no information on the Stokes drift was given for these data, the observations shown in Fig. 11b have been rescaled according to Eq. (3), with $\mathrm{La}_{t}=0.3$, suggesting that the magnitude of the observed $\sigma_{w}^{2} / u_{*}^{2}$ is consistent with Langmuir turbulence for a fully developed sea. The shapes of the observed and modeled profiles are similar, with the maximum variance occurring around $z / h=-0.15$ and -0.2 .

Profiles of $\sigma_{v}^{2} / w^{2} *_{L}$ are shown in Fig. 11c. The variance decreases rapidly with depth between the surface and $z / h=-0.1$, decreasing more gradually over the rest of the mixed layer. Plueddemann et al. (1996) and Smith (1998) estimated $\sigma_{v}$ just below the surface using sidescan Doppler sonar. Estimates of the surface stress and the Stokes drift for these data are provided in Smith (1998). The actual depth of the observations is not well determined and has been set to $2.5 \mathrm{~m}$ in Fig. 11c (Smith 1998). The results from Smith (1998), which are averages over two periods during the same storm, are in reasonable agreement with the LES. In contrast the results from Plueddemann et al. (1996) are about an order of magnitude larger than obtained from the LES. Based on these observations Smith (1999) has suggested that $\sigma_{v} \sim u_{s}$, which is not consistent with the present LES. An analysis similar to that of Smith (1999) has been done by Skyllingstad (2000) and Min and Noh (2004) using LES data, both studies finding that $\sigma_{v} \sim w_{*} *_{L}$.

The depth associated with the sonar observations is in the region where the effects of wave breaking are likely to be important, which is not represented in the present simulations. Min and Noh (2004) and Sullivan et al. (2007) have reported results from two LES studies, which include a parameterization for the effects of breaking waves, in addition to the effects of wave-current interactions. Both studies suggest that the effects of wave breaking on the velocity variance profiles are generally small, except very close to the surface, where TKE was increased. This increase is mainly in the small-scale, subgrid, motions that are probably filtered out of the sonar data. Both Min and Noh (2004) and Sullivan et al. (2007) found that the resolved $\sigma_{v}^{2}$ decreased slightly when wave breaking was included in the simulations, the Langmuir circulations becoming less coherent. 

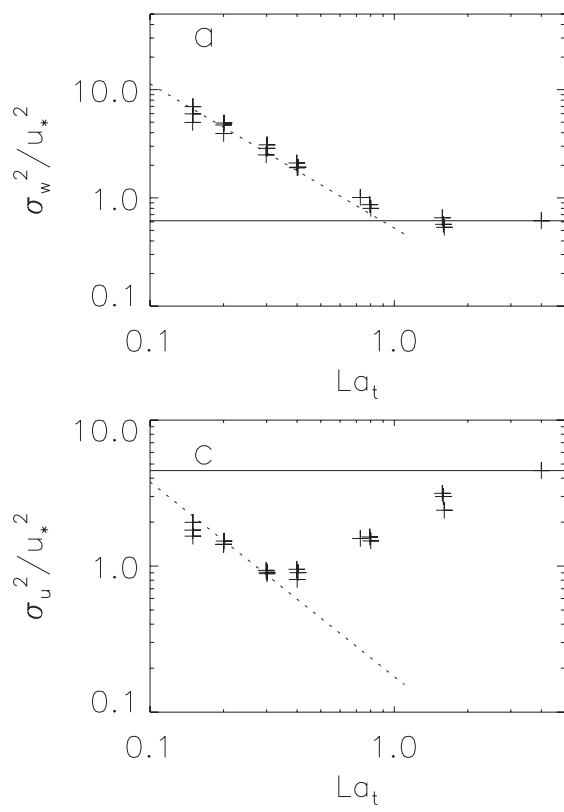

These results suggest that the effects of wave breaking are unlikely to cause the difference between the data in Smith (1999) and the large-eddy simulations.

Figure 11b shows that the depth of the sonar observations lies close to the region where $\sigma_{v}$ decreases rapidly with $z / h$. This suggests that variations in the mixed layer depth could lead to changes in the near surface $\sigma_{v}$ measured by sonar. During the storm studied by Smith (1998) the mixed layer depth appears to have increased, and there were significant variations in mixed layer depth in the Plueddemann et al. (1996) data. It is not clear that these variations in mixed layer depth would lead to the conclusion $\sigma_{v} \sim u_{s}$, but the LES results suggest that the interpretation of the near-surface data in terms of simple scalings is not straightforward.

Profiles of $\sigma_{u}^{2} / w_{* L}^{2}$ are shown in Fig. 11d. In the region close to the surface $\sigma_{u}^{2} / w_{*_{L}}^{2}$ decreases rapidly, reaching a minimum at about $z / h=-0.2$. There is a local maximum in $\sigma_{u}^{2}$, which is not very pronounced, at about $z / h=-0.8$.

Equation (3) implies that the velocity-component variances when nondimensionalised by $u_{*}^{2}$ should vary as $1 / \mathrm{La}_{t}^{4 / 3}$ in the regime dominated by Langmuir turbulence. Figures 12a-c show maximum value of $\sigma_{w}^{2} / u_{*}^{2}$ and the values of $\sigma_{v}^{2} / u_{*}^{2}$ and $\sigma_{v}^{2} / u_{*}^{2}$ at $z / h=-0.2$ (corresponding to the position of the minimum in the $\sigma_{v}^{2}$ profile) as a function of $\mathrm{La}_{t}$. All three variances follow the Langmuir scaling for $\mathrm{La}_{t}<0.5$. This is consistent with $\mathrm{Li}$ et al. (2005) who found that $\sigma_{w}^{2} / u_{*}^{2}$ for Stokesforced simulations increased rapidly with decreasing $\mathrm{La}_{t}$ for $\mathrm{La}_{t}<0.7$. For $\mathrm{La}_{t}>0.5$ both $\sigma_{w}^{2} / u_{*}^{2}$ and $\sigma_{v}^{2} / u_{*}^{2}$ depart from the Langmuir scaling and tend toward values appropriate for shear turbulence.

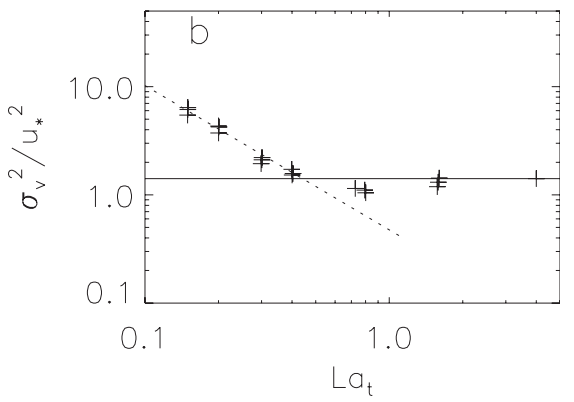

FIG. 12. Variations in variances scaled by $\sigma_{u}^{2}$ with $\mathrm{La}_{t}$. (a) The maximum $\sigma_{w}^{2} / u^{2}$ as a function of $\mathrm{La}_{t}$. The dotted line shows $\sigma_{w}^{2} / u_{*}^{2}=0.53 / \mathrm{La}_{t}^{4 / 3}$. The horizontal line is the value for a neutral shear flow. (b) Shown is $\sigma_{v}^{2} / u_{*}^{2}$ at $z / h=0.2$ as a function of $\mathrm{La}_{t}$. The dotted line shows $\sigma_{v}^{2} / u_{*}^{2}=$ $0.48 / \mathrm{La}_{t}^{4 / 3}$. (c) Shown is $\sigma_{u}^{2} / u_{*}^{2}$ at $z / h=0.2$ as a function of $\mathrm{La}_{t}$. The dotted line shows $\sigma_{u}^{2} / u_{*}^{2}=0.18 / \mathrm{La}_{t}^{4 / 3}$.

The effect of the vortex force is to increase $\sigma_{w}^{2} / u^{2}$ and $\sigma_{v}^{2} / u_{*}^{2}$ above their values in a normal shear flow so that the change from the Langmuir regime to the shear regime appears to be continuous and gradual. The behavior of $\sigma_{u}^{2} / u_{*}^{2}$ is rather different. In the region of the Stokes shear $\sigma_{u}^{2} / u_{*}^{2}$ is reduced below the value for normal shear turbulence. Figure 12 shows that the magnitude of $\sigma_{u}^{2} / u_{*}^{2}$ at $z / h=-0.2$ reaches a minimum around $\mathrm{La}_{t}=0.4$. For smaller values of $\mathrm{La}_{t}, \sigma_{u}^{2} / u_{*}^{2}$ increases according to the Langmuir scaling given by Eq. (3). For values of $\mathrm{La}_{t}$ greater than $0.4, \sigma_{u}^{2} / u_{*}^{2}$ increases toward the value appropriate to shear turbulence, but this is not reached until $\mathrm{La}_{t} \approx 2-3$. The variation of $\sigma_{u}^{2} / u_{*}^{2}$ is similar to that shown by the dissipation rate (Fig. 4) and is also due to the variations in TKE production due to Stokes shear and current shear.

\section{b. The effects of Coriolis-Stokes forcing}

The results presented so far have established that Langmuir turbulence can be defined as a distinct scaling regime, as suggested by the $\mathrm{Li}$ et al. (2005) regime diagram. As explained above this implies that velocity statistics scaled by $w_{*}$ should not be functions of $\mathrm{La}_{t}$, or if scaled by $u *$ should vary with $\mathrm{La}_{t}$ in a way consistent with Eq. (3). Figure 13 shows the minimum value of $\overline{w^{\prime 3}} / u_{*}^{3}$ as a function of $\mathrm{La}_{t}$. In this case the LES results do not follow the dependence on $\mathrm{La}_{t}$, varying somewhat more slowly with $\mathrm{La}_{t}$ than Eq. (3) suggests, implying that $\overline{w^{\prime 3}} / w_{*_{L}}^{3}$ is not constant.

Figure 2 shows that the transport of TKE from the region of Stokes production is mainly associated with $\overline{w^{\prime 3}}$, and the minimum value of $\overline{w^{\prime 3}}$ represents net energy flux 

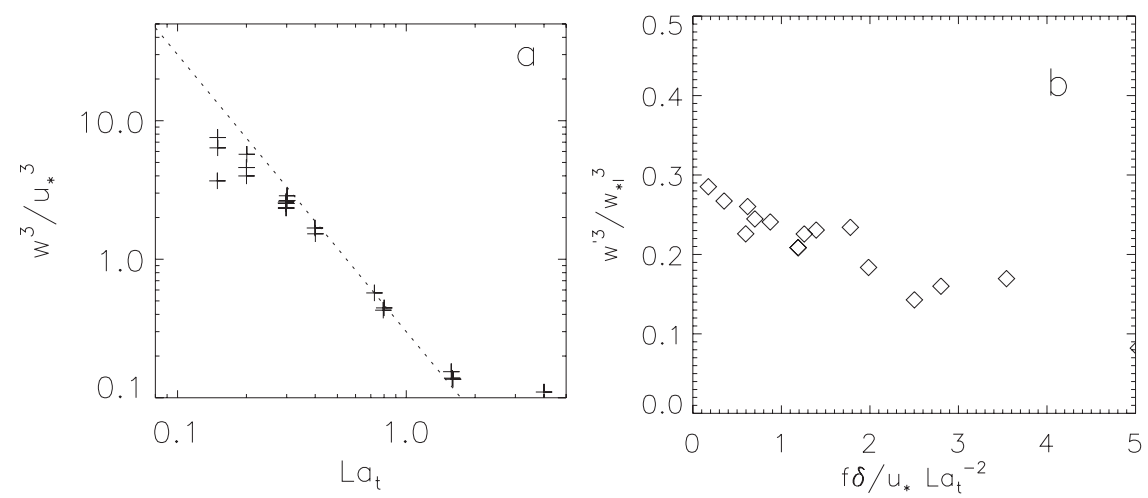

FIG. 13. Plot of the minimum value of $\overline{w^{\prime} 3} / u_{*}^{3}$ as a function of the turbulent Langmuir number, $\mathrm{La}_{t}$. The dotted line shows the variation of $\overline{w^{\prime 3}} / u_{*}^{3}$ expected for Langmuir turbulence. The point at $\mathrm{La}_{t}=4$ is for the shear only simulation. (b) Shown is $\overline{w^{\prime 3}} / u_{*}^{3}$ as a function of $f \delta / u_{*} \mathrm{La}_{t}^{-2}$.

from this region into the bulk of the mixed layer. This suggests that the failure of $\overline{w^{\prime 3}}$ to follow Langmuir scaling may be a consequence of the variation in the Stokes production, noted in section $5 \mathrm{c}$.

The Stokes-shear production at a depth $\delta$ can be estimated by the Taylor expansion as

$$
-\left.\overline{u^{\prime} w^{\prime}} \frac{\partial u_{s}}{\partial z}\right|_{z=-\delta} \approx-\left(\overline{u^{\prime} w_{0}^{\prime}}+\left.\frac{\partial \overline{u^{\prime} w^{\prime}}}{\partial z}\right|_{z=0} \delta\right) \frac{u_{\mathrm{s} 0}}{\delta} .
$$

The stress gradient at the surface varies with $f u_{\mathrm{s} 0}$ (see Fig. 9), so the Stokes production should vary like $f u_{s 0}^{2}$. Assuming that the energy exported from the region of Stokes shear is just a fraction of the Stokes production, then $\overline{w_{b}^{\prime 3}} / \delta \sim f u_{s 0}^{2}$, and consequently $\overline{w_{b}^{\prime 3}} / w_{* L}^{3} \sim f \delta / u * \mathrm{La}_{t}^{-2}$, where $\overline{w_{b}^{\prime 3}}$ is the value of $\overline{w^{\prime 3}}$ at the base of the region of Stokes shear. Figure $13 \mathrm{~b}$ shows $\overline{w_{b}^{\prime 3}} / w_{* L}^{3}$ as a function of $f \delta / u * \mathrm{Lat}_{t}^{-2}$. The magnitude of $\overline{w_{b}^{\prime 3}} / w_{*_{L}}^{3}$ decreases with increasing $f \delta / u * \mathrm{La}_{t}^{-2}$, consistent with the suggestion that the failure of $\overline{w^{\prime 3}}$ to follow Langmuir scaling is due to the Coriolis-Stokes forcing of the mean momentum budget. Because the curvature of the stress profiles increases with decreasing $\mathrm{La}_{t}, \overline{w_{b}^{\prime}} / u_{*}^{3}$ would be expected to increase less rapidly with $\mathrm{La}_{t}$ than Eq. (3) would suggest, which is the case (Fig. 13). The maximum in the vertical velocity variance, $\sigma_{w}^{2} / w_{*}^{2}$, also shows a weak dependence on $f \delta / u^{*} \mathrm{La}_{t}^{-2}$, (not shown), which accounts for some of the variability in the profiles of $\sigma_{w}^{2} / w_{*}^{2}$, apparent in Fig. 11b.

The production of TKE through Stokes shear shows a coupling to the mean momentum budget through the Coriolis-Stokes term, but the total shear production shows much less variation (Fig. 7b). Although production associated with the current shear compensates for the variations in the Stokes production it does not appear to compensate for the variations in $\overline{w^{\prime 3}}$. This is because the transport associated with production of TKE by current shear is due to the fluxes $\overline{w^{\prime} u^{\prime 2}}$ and $\overline{w^{\prime} v^{\prime 2}}$, and is also smaller than that associated with Stokes production (Fig. 2b).

These results highlight a significant difference between Langmuir turbulence, as represented by the CL2 mechanism, and normal shear turbulence. In the simulation of Langmuir turbulence the Stokes drift is imposed upon the turbulence field but is not itself affected by the resulting turbulence. This is different to a normal shear flow in which the mean shear and turbulence are closely linked. This means that the shear production term in the TKE budget can adjust independently of the mean momentum balance. In this case a dependence of the turbulence properties on the Coriolis parameter is not expected. In the present simulations the Stokes shear production cannot adjust in the same way so that the TKE budget and other properties of the turbulence may depend on the Coriolis parameter, as discussed. In reality it is possible that there could be interactions between the wave-induced shear and turbulence leading to the possibility of an adjustment of the Stokes shear, but this is not included in the CL2 mechanism.

\section{c. The effects of varying the Stokes depth scale}

Figures $14 \mathrm{a}-\mathrm{c}$ show the scaled velocity variance profiles from simulations in which $\delta$ was varied. There seem to be only relatively minor variations in the variance profiles, particularly when compared to the variations in Figs. 11. The most systematic variation is in the depth of the minimum in $\sigma_{u}^{2}$ profile (Fig. 14c), which appears to increase as $\delta$ increases. Figure $14 \mathrm{~d}$ shows the profile of $\sigma_{u}^{2} / w_{*}^{2}{ }_{L}$ as a function of $z / \delta$. The depth scaling collapses the individual profiles onto a single profile for depths less than $\delta$ more effectively than using $z / h$ (cf. Fig. 14c).

These results suggest that the parameter $\delta / h$ is not very significant in determining the properties of Langmuir turbulence. However, for the present simulations 

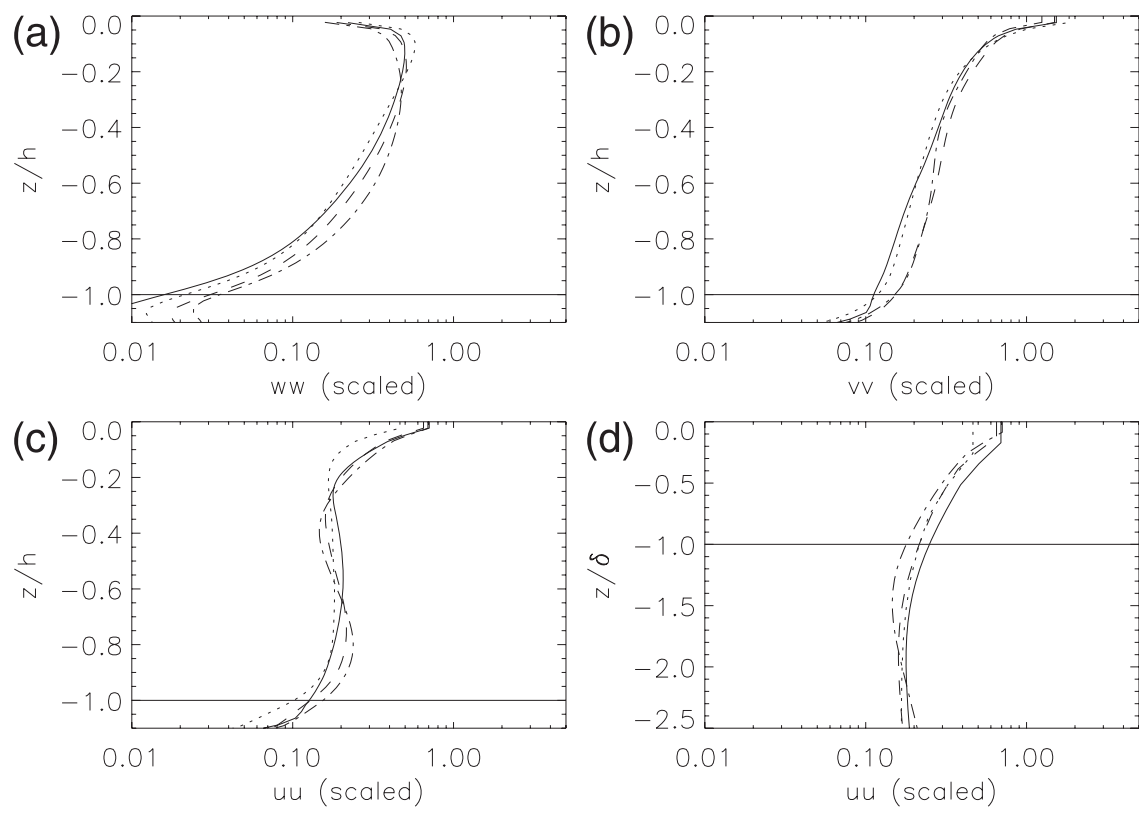

FIG. 14. (a) Profiles of $\sigma_{w}^{2}$ scaled by $w_{*_{L}}^{2}$ for different values of the Stokes depth $\delta$, plotted as a function of $z / h$. The line types are $\delta=3.2 \mathrm{~m}$ (dotted), $\delta=4.8 \mathrm{~m}$ (full), $\delta=6.4 \mathrm{~m}$ (dashed), and $\delta=9.5 \mathrm{~m}$ (dot-dash). (b) As in (a), but for $\sigma_{v}^{2}$. (c) As in (a), but for $\sigma_{u}^{2}$. (d) As in (a), but profiles of $\sigma_{u}^{2}$ scaled by $w_{* L}^{2}$ against nondimensional height $z / \delta$.

$\delta / h$ varies from 0.09 to 0.27 . This lack of variation with changes in $\delta / h$ is consistent with the findings of Harcourt and D'Asaro (2008), who found a strong dependence of $\sigma_{w}^{2} / u_{*}^{2}$ on $h / \delta$ for $h / \delta<0.1$.

\section{Entrainment}

The importance of Langmuir turbulence in deepening the mixed layer is still not clear (Thorpe 2004). Langmuir (1938) suggested that the circulations played a role in the formation of the thermocline. Li et al. (1995) identified periods of mixed layer deepening during the Long-Term Upper Ocean Study (LOTUS) that satisfied the criterion derived in $\mathrm{Li}$ and Garret (1995) for deepening by Langmuir circulations.

Figures 15a-d show the buoyancy, shear, and shear + transport terms in the TKE budget around the base of the mixed layer, with the different panels showing results for different Langmuir numbers. For the highest Langmuir numbers $\left(\mathrm{La}_{t}=0.4-0.3\right)$ current shear is smaller than the transport term as a source of TKE in the thermocline, but as $\mathrm{La}_{t}$ decreases the current shear becomes larger than the transport. This is due in part to an increase in the shear production, and in part a decrease in the transport term. The decrease in the transport term is related to the effects of the CoriolisStokes forcing discussed previously. The buoyancy work in these simulations is about $50 \%$ of the combined shear production and transport of TKE into the thermocline and is comparable to the dissipation rate. Close inspection of Figs. 15a-d also reveals some variation in nondimensional dissipation rate at the base of the mixed layer that appears to be associated with the variations in other terms in the budget. This is responsible for at least part of the variability noted in section 5a.

Figure 16a shows the variation of the entrainment flux with the TKE budget scale $w_{*}^{*}{ }_{L}^{3} / h$, for simulations with $\mathrm{La}_{t}<0.5$. This linear variation of the entrainment flux with $w_{*_{L}}^{3} / h$, indicates that the entrainment is being driven by the Langmuir turbulence by a combination of the transport of TKE and current shear. This contrasts with convectively driven turbulence in which current shear does not play a fundamental role in entrainment. Figure $16 \mathrm{~b}$ shows the entrainment flux, nondimensionalized by $u_{*}^{3} / h$ as a function of $\mathrm{La}_{t}$. For $\mathrm{La}_{t}<0.5$ the nondimensional entrainment flux varies as $1 / \mathrm{La}_{t}^{2}$, consistent with Langmuir turbulence. For larger values of $\mathrm{La}_{t}$ the entrainment rate tends toward the value obtained for a shear-driven mixed layer without wave effects. In these simulations the wave effects lead to a significant enhancement in entrainment relative to the shear-only simulation.

The subgrid contribution to the buoyancy flux is small, even at the base of the mixed layer and in the stable region immediately below, which suggests that the energetics of the entrainment process are being adequately resolved in these simulations. This is also suggested by the magnitude of the dissipation length scale immediately below the base 

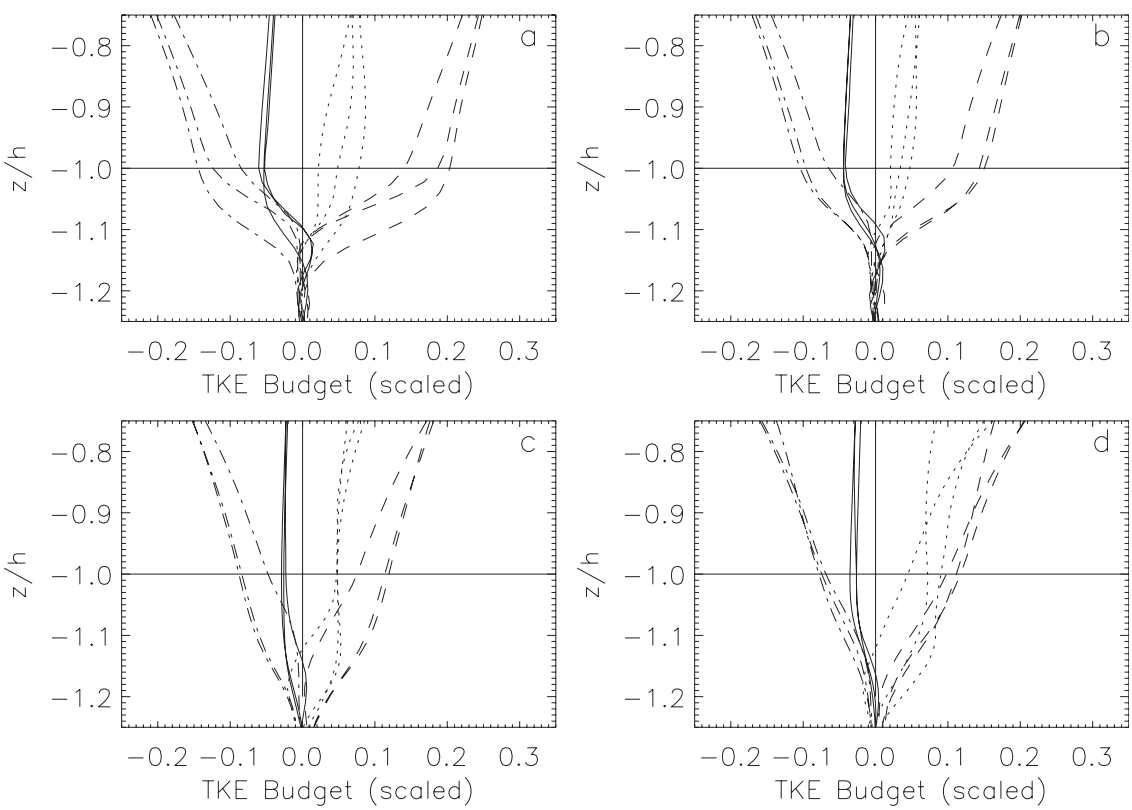

FIG. 15. Plots showing terms in the TKE budget in the lower part of the mixed layer and the thermocline. All profiles are scaled by $w_{* L}^{3} / h$. Buoyancy (full curves), shear production + transport (dashed curves), shear production (dotted curves), dissipation (dash-dot curves). Simulations with (a) $\mathrm{La}_{t}=0.4$, (b) $\mathrm{La}_{t}=0.3$, (c) $\mathrm{La}_{t}=0.2$, and (d) $\mathrm{La}_{t}=0.15$.

of the mixed layer, which is similar to the mixed layer value, and is much larger than the grid scale. At greater depths the dissipation length scale increases, possibly indicating the presence of wave motions.

The Ozmidov scale is smaller than the grid size, so the LES does not resolve the inertial subrange in the stable region. This suggests that while the energetics of the entrainment process is being resolved the details of the process are probably not. The mean structure in the entrainment region may depend on details of the entrainment process and hence on the resolution of the LES (Sullivan et al. 1998).
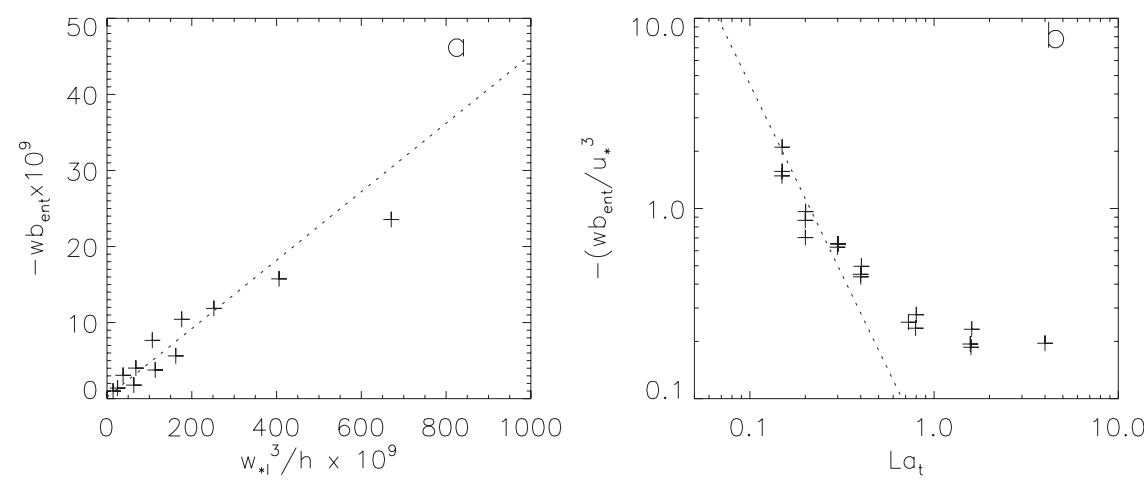

FIG. 16. Plot of the entrainment buoyancy flux, $\overline{w^{\prime} b^{\prime}}$ ent , as a function of $w_{*_{L}}^{3} / h$ for simulations with $\mathrm{La}_{t}<0.5 \overline{w^{\prime} b^{\prime}}$ ent (dotted curve). (b) The entrainment buoyancy flux, normalized by $u_{*}^{3} / h$, as a function of $\mathrm{La}_{t}$. Here $\overline{w^{\prime} b^{\prime}}{ }_{\text {ent }} h / u_{*}^{3}=0.045 / \mathrm{La}_{t}^{2}$ (dotted line). 
spectra, restricting the range of parameter space covered by realistic variations in winds and wave fields.

The second idealization uses the ability of the LES technique to isolate processes that in reality would be difficult to separate, such as Langmuir turbulence and wave breaking. The LES is being used as a laboratory version of the oceanic mixed layer in which controlled experiments can be carried out.

In addition to the idealized nature of the simulations, large-eddy simulations make a number of assumptions. In common with previous studies of Langmuir turbulence this study assumes that the effects of waves on mixed layer turbulence can be represented through the CraikLeibovich (CL2) model. There are also uncertainties associated with the subgrid model as well as resolution and numerical approximations.

Variations in the magnitude of the dissipation rate relative to that expected for shear-driven turbulence and the results for $\sigma_{w}^{2} / u_{*}^{2}$ of D'Asaro (2001) and Tseng and D'Asaro (2004) suggest that turbulence in the ocean mixed layer is not purely shear driven. Although comparison with LES is suggestive of Langmuir turbulence it is not conclusive, and also given the uncertainties in LES a more rigorous testing of the scaling results presented here, against observations, is clearly desirable.

To test the proposed scaling it would be necessary to obtain data over a range of sea states to obtain a reasonable variation in $\mathrm{La}_{t}$. Harcourt and D'Asaro (2008) calculated $\mathrm{La}_{t}$ for a range of wind speeds and wave ages for pure wind-driven seas using empirical wave spectra and drag coefficients. Considering wind speeds up to $15 \mathrm{~m} \mathrm{~s}^{-1}$, their Table 1 suggests a variation in $\mathrm{La}_{t}$ from 0.27 to 0.36 , for wave ages, $c_{p} / U_{10}$ (where $c_{p}$ is the phase speed at the spectral at the peak of the wave spectrum and $U_{10}$ is the $10-\mathrm{m}$ wind speed) varying from 1.2 to 0.6 and $U_{10}$ varying from 8 to $15 \mathrm{~m} \mathrm{~s}^{-1}$.

If the proposed scaling is valid Eq. (3) suggests that over this range of $\mathrm{La}_{t}, \epsilon h / u_{*}^{3}$ should vary by a factor of 1.8 , and $\sigma_{w}^{2} / u_{*}^{2}$ by a factor of 1.5 . Smaller ranges of $\mathrm{La}_{t}$ would produce correspondingly smaller variations in dissipation and vertical velocity variance making it harder to test the scaling. To obtain the required variations in wind and wave condition observations would probably need to be obtained during midlatitude storms to cover the range of $\mathrm{La}_{t}$. The results of Harcourt and D'Asaro (2008) suggest that $\delta / h$ should also be greater than 0.1 , putting constraints on the mixed layer depth. Of course, in addition to the turbulence measurements, measurements of the wave field to estimate the Stokes drift are also required.

The above gives an idea of the data needed to test the LES predictions quantitatively. However, to translate this into an observational strategy and to assess the feasibility of obtaining sufficient data would need a careful consideration of the errors in measuring dissipation (both instrumental and sampling) in the oceanic mixed layer.

\section{Conclusions}

Large-eddy simulations have been used to characterize Langmuir turbulence in the oceanic mixed layer. The main results of this study are as follows:

- The velocity and length scales of Langmuir turbulence are $w_{* L}=\left(u_{*}^{2} u_{s 0}\right)^{1 / 3}$ and the mixed layer depth.

- When the turbulent Langmuir number is less than 0.5 profiles of dissipation rate and velocity variances scale with $w_{* L}$ and are independent of $\mathrm{La}_{t}$. Here $\mathrm{La}_{t}<0.5$ corresponds to the Langmuir turbulence regime, which is distinct from normal shear-driven turbulence.

- The buoyancy flux at the base of the mixed layer scales with $w_{*}^{3} / h$ entrainment by Langmuir turbulence is larger than for normal shear-driven turbulence.

- The properties of Langmuir turbulence depend only weakly on the Stokes depth $\delta$ for the range of $\delta / h$ considered in this study.

- The transition between Langmuir turbulence and shear turbulence occurs for $0.5<\mathrm{La}_{t}<2$.

- The effect of the Coriolis force on the Stokes drift in the mean momentum budget leads to a coupling between the TKE budget and the mean momentum budget. One effect of this coupling is that turbulent transport of TKE, and to a lesser extent the variance of the vertical component of the turbulent velocity, depend on the Coriolis parameter. This effect is most significant for small values of $\mathrm{La}_{t}$.

- Currently available observations cannot be used to test the LES scaling because the necessary wave parameters are not usually available from published sources.

Acknowledgments. This work was supported by the Natural Environment Research Council (NERC) Research Grant NE/DO10810/1.

\section{REFERENCES}

Agrawal, Y. C., E. A. Terray, M. A. Donelan, P. A. Hwang, A. J. Williams, W. M. Drennan, K. K. Kahma, and S. A. Kitaigorodskii, 1992: Enhanced dissipation of kinetic energy beneath breaking waves. Nature, 359, 219-220.

Anis, A., and J. N. Moum, 1992: The superadiabatic surface layer in the ocean during convection. J. Phys. Oceanogr., 22, 1221-1227.

Brown, A. R., S. H. Derbyshire, and P. J. Mason, 1994: Large-eddy simulation of stable atmpospheric boundary layers with a revised stochastic subgrid model. Quart. J. Roy. Meteor. Soc., 120, $1485-1512$.

— M. K. MacVean, and P. J. Mason, 2000: The effects of numerical dissipation in large eddy simulations. J. Atmos. Sci., 57, 3337-3348. 
Bryden, D., S. Sun, and R. Bleck, 1999: A new approximation of the equation of state for seawater, suitable for numerical ocean models. J. Geophys. Res., 104, 1537-1540.

Craig, P. D., and M. L. Banner, 1994: Modeling wave-enhanced turbulence in the ocean surface layer. J. Phys. Oceanogr., 24, 2546-2559.

Craik, A. D. D., and S. Leibovich, 1976: A rational model for Langmuir circulations. J. Fluid Mech., 73, 401-426.

Csanady, G. T., 1997: The slip law of the free surface. J. Oceanogr., 53, 67-80.

D'Asaro, E. A., 2001: Turbulent vertical kinetic energy in the ocean mixed layer. J. Phys. Oceanogr., 31, 3530-3537.

Grant, A. L. M., 1992: The structure of turbulence in the nearneutral atmospheric boundary layer. J. Atmos. Sci., 49, 226-239.

Greenan, B. J. W., N. S. Oakey, and F. W. Dobson, 2001: Estimates of dissipation in the ocean mixed layer using a quasi-horizontal microstructure profiler. J. Phys. Oceanogr., 31, 992-1004.

Harcourt, R. R., and E. A. D'Asaro, 2008: Large-eddy simulation of Langmuir turbulence in pure wind driven seas. J. Phys. Oceanogr., 38, 1542-1562.

Holtslag, A. A. M., and F. T. M. Nieuwstadt, 1986: Scaling the atmospheric boundary layer. Bound.-Layer Meteor., 36, 201-209.

— , and C. H. Moeng, 1991: Eddy diffusivity and countergradient transport in the convective atmospheric boundary layer J. Atmos. Sci., 48, 1690-1698.

Hunt, J. C. R., and J. M. R. Graham, 1978: Free stream turbulence near plane boundaries. J. Fluid Mech., 84, 209-235.

Kitaigorodskii, S. A., and J. L. Lumley, 1983: Wave-turbulence interactions in the upper ocean. Part I: The energy balance of the interacting fields of surface wind waves and wind-induced threedimensional turbulence. J. Phys. Oceanogr., 13, 1977-1987.

Langmuir, I., 1938: Surface motion of water induced by wind. Science, 38, 119-123.

Large, W. G., J. C. McWilliams, and S. C. Doney, 1994: Oceanic vertical mixing: A review and a model with a nonlocal boundary layer parameterization. Rev. Geophys., 32, 363-403.

Li, M., and C. Garret, 1995: Is Langmuir circulation driven by surface waves or surface cooling. J. Phys. Oceanogr., 25, 64-76.

$\ldots$, K. Zahariev, and C. Garret, 1995: Role of Langmuir circulation in the deepening of the ocean surface mixed layer. Science, 270, 1955-1957.

_ - C. Garrett, and E. Skyllingstad, 2005: A regime diagram for classifying turbulent large eddies in the upper ocean. Deep Sea Res. I, 52, 259-278.

Lombardo, C. P., and M. C. Gregg, 1989: Similarity scaling of viscous and thermal dissipation in a convecting surface boundary layer. J. Geophys. Res., 94, 6273-6284.

Lozovatsky, I. D., E. Roget, H. J. S. Fernando, M. Figueroa, and S. Shapovalov, 2006: Sheared turbulence in a weakly stratified upper ocean. Deep-Sea Res. I, 53, 387-407.

McWilliams, J. C., and P. P. Sullivan, 2000: Vertical mixing by Langmuir circulations. Spill Sci. Technol., 6, 225-237.

- — - and C. H. Moeng, 1997: Langmuir turbulence in the ocean. J. Fluid Mech., 334, 1-30.

Min, H. S., and Y. Noh, 2004: Influence of the surface heating on Langmuir circulation. J. Phys. Oceanogr., 34, 2630-2641.

Moeng, C. H., and P. P. Sullivan, 1991: A comparison of shear- and buoyancy-driven planetary boundary layer flows. J. Atmos. Sci., 48, 999-1022.

Noh, Y., and H. S. Min, 2004: Large eddy simulation of the ocean mixed layer: The effects of wave breaking and Langmuir circulation. J. Phys. Oceanogr., 34, 720-735.
Piacsek, S. A., and G. P. Williams, 1970: Conservation properties of convection difference schemes. J. Comput. Phys., 6, 392-405.

Plueddemann, A. J., J. A. Smith, D. M. Farmer, R. A. Weller, W. R. Weller, R. Pinkel, S. Vagel, and A. Gnanadesikan, 1996: Structure and variability of Langmuir circulation during the surface waves processes program. J. Geophys. Res., 101, $3525-3543$

Polton, J. A., and S. E. Belcher, 2007: Langmuir turbulence and deeply penetrating jets in an unstratified mixed layer. J. Geophys. Res., 112, C09020, doi:10.1029/2007JC004205.

D. M. Lewis, and S. E. Belcher, 2005: The role of waveinduced Coriolis-Stokes forcing on the wind-driven mixed layer. J. Phys. Oceanogr., 35, 444-457.

Shutts, G. J., and M. E. B. Gray, 1994: A numerical modelling study of the geostrophic adjustment process following deep convection. Quart. J. Roy. Meteor. Soc., 120, 1145-1178.

Skyllingstad, E. D., 2000: Scales of Langmuir circulation generated using a large-eddy simulation model. Spill Sci. Technol., 6, 239-246.

- and D. W. Denbo, 1995: An ocean large-eddy simulation of Langmuir circulations and convection in the surface mixed layer. J. Geophys. Res., 100, 8501-8522.

Smagorinsky, J., 1963: General circulation experiments with the primitive equations. Part I: The basic experiment. Mon. Wea. Rev., 91, 99-164.

Smith, J. A., 1996: Observations of Langmuir circulation, waves and the mixed layer. Air-Sea Interface: Radio and Acoustic Semsing, Turbulence, and Wave Dynamics, M. A. Donelan, W. H. Hi, and W. J. Plant, Eds., University of Toronto Press, 613-622. 1998: Evolution of Langmuir circulation during a storm. J. Geophys. Res., 103, 12 649-12 668.

1999: Observations of wind, waves and the mixed layer: The scaling of surface motions. The Wind-Driven Air-Sea Interface, M. L. Banner, Ed., The University of New South Wales, 231-238.

Smyth, W. D., E. D. Skyllingstad, G. B. Crawford, and H. Wijesekera, 2002: Nonlocal fluxes and Stokes drift effects in the k-profile parameterization. Ocean Dyn., 52, 104-115.

Sullivan, P. P., C.-H. Moeng, B. Stevens, D. H. Lenschow, and S. D. Mayor, 1998: Structure of the entrainment zone capping the convective atmospheric boundary layer. J. Atmos. Sci., 55, 3042-3064.

, J. C. McWilliams, and W. K. Melville, 2007: Surface gravity wave effects in the oceanic boundary layer: Large-eddy simulation with vortex force and stochastic breakers. J. Fluid Mech., 593, 405-452.

Teixeira, M. A. C., and S. E. Belcher, 2002: On the distortion of turbulence by a progressive surface wave. J. Fluid Mech., 458, 229-267.

Tennekes, H., and J. L. Lumley, 1972: A First Course in Turbulence. Cambridge University Press, 372 pp.

Terray, E. A., M. A. Donelan, Y. C. Agrawal, W. M. Drennan, K. K. Kahma, A. J. Williams III, P. A. Hwang, and S. A. Kitaigorodskii, 1996: Estimates of kinetic energy dissipation under breaking waves. J. Phys. Oceanogr., 26, 792-807.

Thorpe, S. A., 2004: Langmuir circulation. Annu. Rev. Fluid, 36, 55-79.

, T. R. Osborne, J. F. E. Jackson, A. J. Hall, and R. G. Lueck, 2003: Measurements of turbulence in the upper-ocean mixing layer using autosub. J. Phys. Oceanogr., 33, 122-145.

Tseng, R. S., and E. A. D'Asaro, 2004: Measurements of turbulent vertical kinetic energy in the ocean mixed layer from Langrangian floats. J. Phys. Oceanogr., 34, 1984-1990. 